\title{
Imido titanium ethylene polymerization catalysts containing triazacyclic ligands
}

Nico Adams, Henricus J. Arts, Paul D. Bolton, Dan Cowell, Stuart R. Dubberley, Nic. Friederichs, Craig M. Grant, Mirko Kranenburg, Andrew J. Sealey, Bing Wang,

Paul J. Wilson, Martin Zuideveld, Alexander J. Blake, Martin Schröder and Philip Mountford*

\section{Supporting information}

Further representations of the crystal structure of $\mathrm{Ti}\left(\mathrm{NC}_{6} \mathrm{~F}_{5}\right)\left(\mathrm{Me}_{3}[9] \mathrm{aneN}_{3}\right) \mathrm{Cl}_{2}(\mathbf{5})$

Further details of the polystyrene-supported catalyst systems

Further details of the $\mathrm{R}_{3}[6] \mathrm{aneN}_{3}$-supported catalyst systems and their $\mathrm{X}$-ray structures.

Experimental details for the polystyrene-supported and $\mathrm{R}_{3}[6]$ ane $\mathrm{N}_{3}$-supported compounds

Library characterization, yields of compounds and polymerization yields and activities. 




Figure S1. Displacement ellipsoid plot $(20 \%)$ of $\mathrm{Ti}\left(\mathrm{NC}_{6} \mathrm{~F}_{5}\right)\left(\mathrm{Me}_{3}[9] \mathrm{aneN}_{3}\right) \mathrm{Cl}_{2}(\mathbf{5}) . \mathrm{H}$ atoms omitted.



Figure S2. Relationship between the $\mathrm{C}_{6} \mathrm{~F}_{5}$ groups associated with $\mathrm{Ti}(1)$ and $\mathrm{Ti}(1 \mathrm{~B})$ in molecules of $\mathrm{Ti}\left(\mathrm{NC}_{6} \mathrm{~F}_{5}\right)\left(\mathrm{Me}_{3}[9] \mathrm{aneN}_{3}\right) \mathrm{Cl}_{2}(\mathbf{5})$. View is projected strictly onto the $\{\mathrm{C}(10), \mathrm{C}(11), \mathrm{C}(12), \mathrm{C}(13)$, $\mathrm{C}(14), \mathrm{C}(15)\}$ least-squares plane. $\mathrm{H}$ atoms omitted and all atoms drawn as spheres of arbitrary radius. Symmetry operator: $\mathrm{B},[2-x,-y, 1-z]$. 


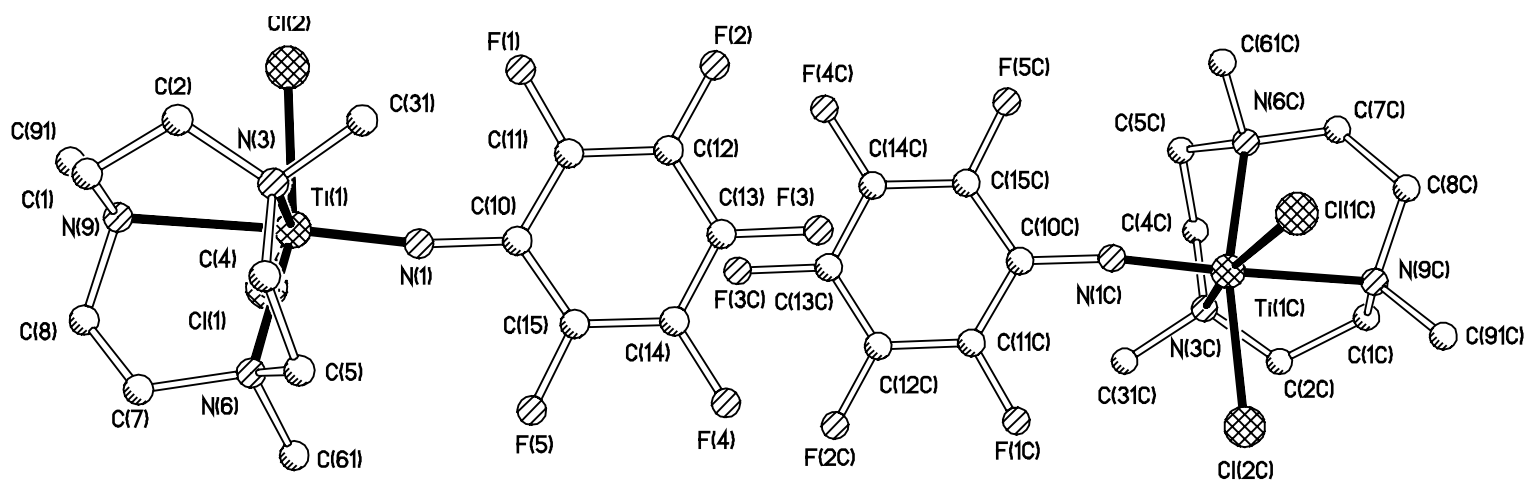

Figure S3. Relationship between the $\mathrm{C}_{6} \mathrm{~F}_{5}$ groups associated with $\operatorname{Ti}(1)$ and $\operatorname{Ti}(1 \mathrm{C})$ in molecules of $\mathrm{Ti}\left(\mathrm{NC}_{6} \mathrm{~F}_{5}\right)\left(\mathrm{Me}_{3}[9] \mathrm{aneN}_{3}\right) \mathrm{Cl}_{2}(\mathbf{5})$. View is projected strictly onto the $\{\mathrm{C}(10), \mathrm{C}(11), \mathrm{C}(12), \mathrm{C}(13)$, $\mathrm{C}(14), \mathrm{C}(15)\}$ least-squares plane. $\mathrm{H}$ atoms omitted and all atoms drawn as spheres of arbitrary radius. Symmetry operator: $\mathrm{C},[2-x,-1-y, 1-z]$.

\section{Polystyrene-supported precatalysts.}

For immobilization via the imido ligand we used our previously reported ${ }^{1}$ synthon $\mathrm{Ti}\left(\mathrm{NCH}_{2} \mathrm{PS}\right) \mathrm{Cl}_{2}\left(\mathrm{NHMe}_{2}\right)_{2}$ where "PS" represents $1 \%$ cross-linked polystyrene.
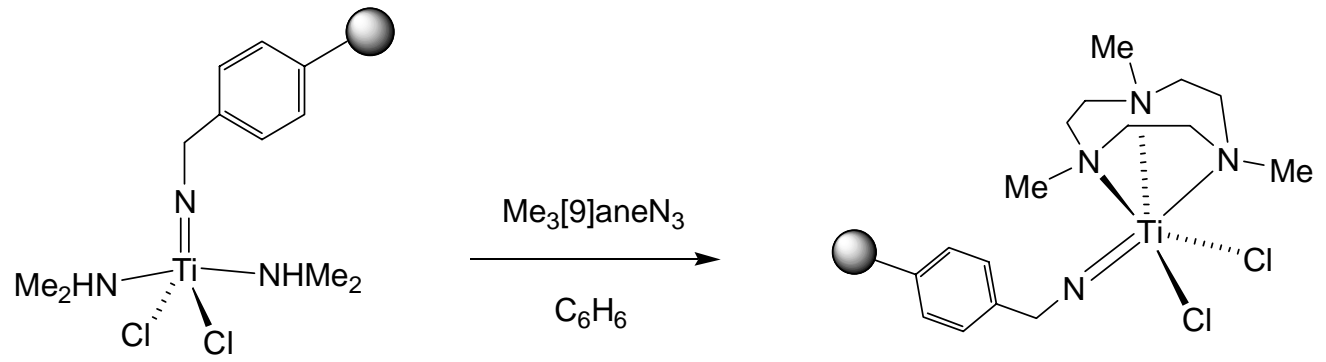

7

As summarized above, shaking (to avoid mechanical damage) a pre-swollen sample of the dark red resin with 1.5 equivs. $\mathrm{Me}_{3}[9] \operatorname{aneN}_{3}\left(50 \%\right.$ excess) in $\mathrm{C}_{6} \mathrm{H}_{6}$ for 16 hours led to a gradual decolorization, and after filtration, washing and drying the pale cream resin $\mathrm{Ti}\left(\mathrm{NCH}_{2} \mathrm{PS}\right)\left(\mathrm{Me}_{3}[9] \mathrm{aneN}_{3}\right) \mathrm{Cl}_{2}$ (7) was obtained. The colour of this material is reminiscent of that of the molecular analog $\mathrm{Ti}\left(\mathrm{NCH}_{2} \mathrm{Ph}\right)\left(\mathrm{Me}_{3}[9] \mathrm{aneN}_{3}\right) \mathrm{Cl}_{2}(\mathbf{6})$. The resin 7 was characterized by $\mathrm{IR}$ and ${ }^{1} \mathrm{H}$ and ${ }^{13} \mathrm{C}-\left\{{ }^{1} \mathrm{H}\right\}$ gel-phase MAS - NMR spectroscopy. The IR spectrum showed that the sharp and distinctive $\mathrm{v}(\mathrm{N}-\mathrm{H})$ absorption of $\mathrm{Ti}\left(\mathrm{NCH}_{2} \mathrm{PS}\right) \mathrm{Cl}_{2}\left(\mathrm{NHMe}_{2}\right)_{2}\left(3268 \mathrm{~cm}^{-1}\right)$ was absent, consistent with displacement of the $\mathrm{NHMe}_{2}$ ligands. The NMR spectra showed no resonances for $\mathrm{NHMe}_{2}$ or $\mathrm{NHMe}_{2}$ groups, while resonances for a bound $\mathrm{Me}_{3}[9] \mathrm{aneN}_{3}$ ligand and new $\underline{\mathrm{CH}}_{2} \mathrm{PS}$ linkage were clearly discernable. Regrettably, as was also the case for the synthon $\mathrm{Ti}\left(\mathrm{NCH}_{2} \mathrm{PS}\right) \mathrm{Cl}_{2}\left(\mathrm{NHMe}_{2}\right)_{2},{ }^{1}$ elemental analysis of 7 repeatedly returned lower than expected $\% \mathrm{~N}, \mathrm{Cl}$ and $\mathrm{Ti}$ values. This is attributed to incomplete combustion or acid digestion of the metal-containing resin. However, on 
the basis of its spectroscopic data (and the behavior of the solution phase analogs) we are confident of the quantitative formation of 7.

Scheme S1 summarizes the synthesis of a new triazacyclononane-functionalized polystyrene resin $(\mathbf{8})$ and the subsequent formation of another solid phase-supported imido titanium pre-catalyst. Supported triazacyclononanes and their complexes with divalent later transition metal ions and $\mathrm{Hg}^{2+}$ have been reported previously. ${ }^{2-6}$ Reaction of the lithiated macrocycle $\mathrm{LiMe}_{2}[9] \mathrm{aneN}_{3}{ }^{7}$ with preswollen commercial $1 \%$ cross-linked chloromethyl polystyrene in THF gave a deep burgundy coloured solid (color possibly due to trace radical anion side-products). Washing/quenching with THF, EtOH and $\mathrm{Et}_{2} \mathrm{O}$ afforded a very pale yellow resin (8). Elemental analysis was consistent with the absence of residual chlorine and (based on \% N) suggested a macrocycle loading of 1.51 mmol. $\mathrm{g}^{-1}$ which is comparable to the $1.60 \mathrm{mmol} . \mathrm{g}^{-1}$ functional group loading of the starting resin. The ${ }^{1} \mathrm{H}$ and ${ }^{13} \mathrm{C}$ MAS NMR spectra of $\mathbf{8}$ were fully consistent with the proposed product.

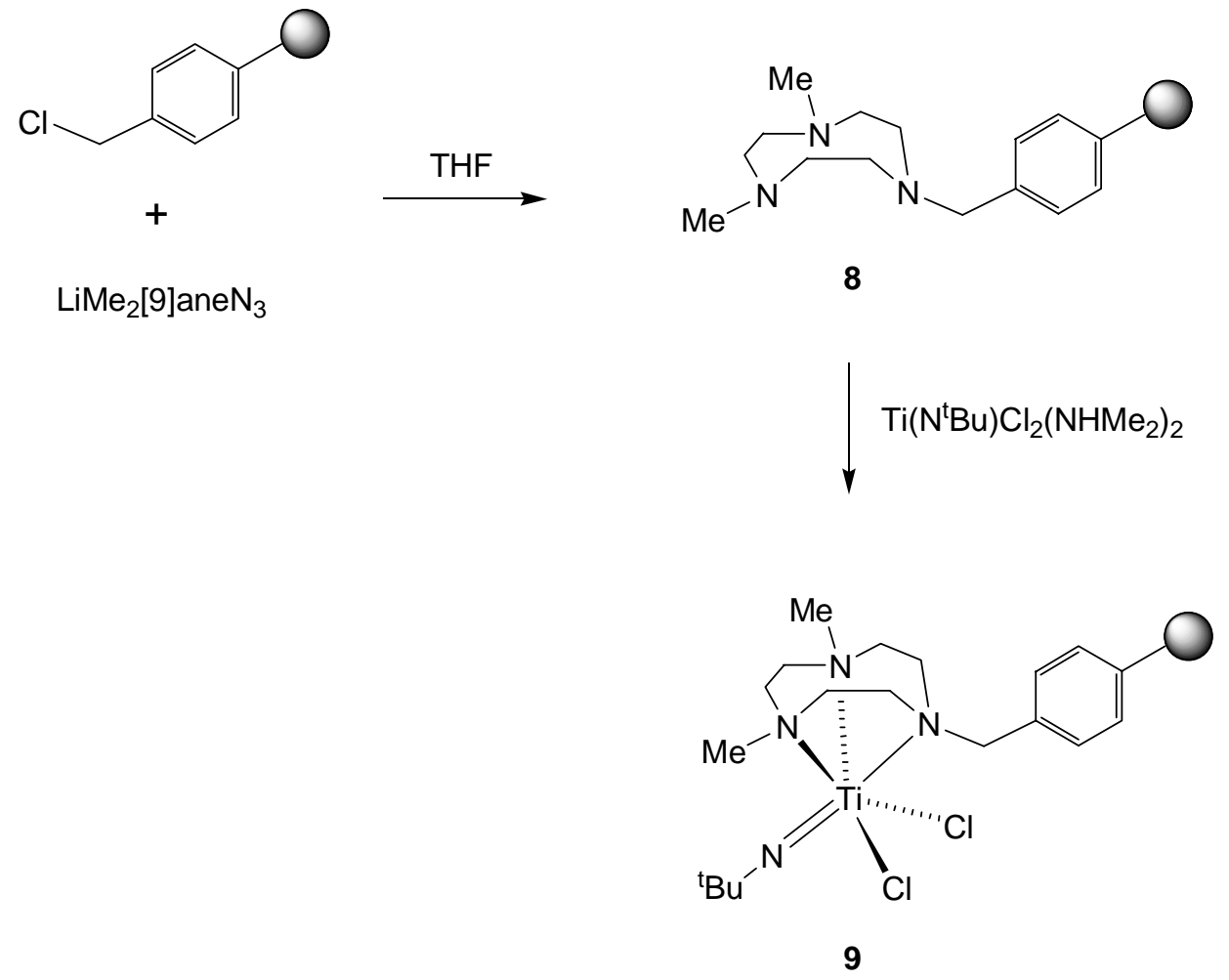

Scheme S1. Synthesis of $\mathrm{PSCH}_{2} \mathrm{Me}_{2}[9] \mathrm{aneN}_{3}(\mathbf{8})$ and $\mathrm{Ti}\left(\mathrm{N}^{\mathrm{t} B u}\right)\left(\mathrm{PSCH}_{2} \mathrm{Me}_{2}[9] \operatorname{aneN}_{3}\right) \mathrm{Cl}_{2}(\mathbf{9})$

Subsequent reaction of pre-swollen $\mathrm{PSCH}_{2} \mathrm{Me}_{2}[9] \operatorname{aneN}_{3}(\mathbf{8})$ with the tert-butyl imido synthon ${ }^{1}$ $\mathrm{Ti}\left(\mathrm{N}^{\mathrm{t}} \mathrm{Bu}\right) \mathrm{Cl}_{2}\left(\mathrm{NHMe}_{2}\right)_{2}$ in $\mathrm{C}_{6} \mathrm{H}_{6}$ led to a gradual decolorization of the solution phase as the metal was complexed to the resin, which was in turn isolated to give pale orange $\mathrm{Ti}\left(\mathrm{N}^{\mathrm{t}} \mathrm{Bu}\right)\left(\mathrm{PSCH}_{2} \mathrm{Me}_{2}[9] \mathrm{aneN}_{3}\right) \mathrm{Cl}_{2}(\mathbf{9})$. The originally well-swollen resin 8 contracted significantly (as judged by eye) during the reaction. The ${ }^{1} \mathrm{H}$ and ${ }^{13} \mathrm{C}$ MAS NMR spectra of 9 (re-swollen in $\mathrm{CD}_{2} \mathrm{Cl}_{2}$ ) showed none of the resonances for $\mathbf{8}$ in the same solvent, and although the spectra were rather broad they were in accord with the structure proposed in Scheme 2. The spectra indicated the Supporting Information page S4 
presence of a single major isomer, although an alternative one with the $\mathrm{CH}_{2} \mathrm{PS}$ substituent cis to the $\mathrm{Ti}=\mathrm{N}^{\mathrm{t}} \mathrm{Bu}$ ligand is in principle possible ${ }^{8}$ and may be present in small amounts.

\section{Triazacyclohexane-supported imido titanium pre-catalysts.}

We prepared $\mathrm{Ti}(\mathrm{NR})\left(\mathrm{Me}_{3}[6] \mathrm{aneN}_{3}\right) \mathrm{Cl}_{2}\left(\mathrm{R}=\mathrm{Ph}(\mathbf{1 2}), \mathrm{C}_{6} \mathrm{~F}_{5}(\mathbf{1 3})\right)$ in 69 and $93 \%$ yields in a "onepot" synthesis starting from $\mathrm{Ti}\left(\mathrm{NMe}_{2}\right)_{2} \mathrm{Cl}_{2}$ followed by the sequential addition of the appropriate aniline and $\mathrm{Me}_{3}[6] \mathrm{aneN}_{3}$. The X-ray structures of these compounds have been determined. So as to make a more thorough comparison with structures of the triazacyclononane series $\mathbf{1}-\mathbf{5}$ we also determined the X-ray structure of the previously reported 11. We also prepared $\mathrm{Ti}(\mathrm{NR})\left(\mathrm{dd}_{3}[6] \mathrm{aneN}_{3}\right) \mathrm{Cl}_{2}\left(\mathrm{R}={ }^{\mathrm{t}} \mathrm{Bu}(\mathbf{1 4}), 2,6-\mathrm{C}_{6} \mathrm{H}_{3}{ }^{\mathrm{i}} \operatorname{Pr}_{2}(\mathbf{1 5})\right)$. These compounds exist as waxy solids and could not be crystallized. The solution NMR spectra of the new compounds $\mathbf{1 2}-\mathbf{1 5}$ were analogous to those of $\mathbf{1 0}$ and $\mathbf{1 1} .^{9}$

$X$-ray crystal structures of $\mathrm{Ti}(\mathrm{NR})\left(\mathrm{Me}_{3}[6] \mathrm{aneN}_{3}\right) \mathrm{Cl}_{2}$. The molecular structures of $\mathrm{Ti}(\mathrm{NR})\left(\mathrm{Me}_{3}[6] \mathrm{aneN}_{3}\right) \mathrm{Cl}_{2}\left(\mathrm{R}=2,6-\mathrm{C}_{6} \mathrm{H}_{3}{ }^{\mathrm{i}} \mathrm{Pr}_{2}\right.$ (11), $\mathrm{Ph}$ (12, two crystallographically independent molecules), $\mathrm{C}_{6} \mathrm{~F}_{5}(\mathbf{1 3})$ ) are shown in Fig. $\mathrm{S} 4$ and selected bond distances are listed in Table $\mathrm{S} 1$ along with those of $\mathrm{Ti}\left(\mathrm{N}^{\mathrm{t}} \mathrm{Bu}\right)\left(\mathrm{Me}_{3}[6] \mathrm{aneN}_{3}\right) \mathrm{Cl}_{2}(\mathbf{1 0})^{9}$ for ease of comparison. Data collection and processing parameters are given in Table S2. As for the larger ring systems $\mathrm{Ti}(\mathrm{NR})\left(\mathrm{Me}_{3}[9] \mathrm{aneN}_{3}\right) \mathrm{Cl}_{2}(\mathbf{1}-\mathbf{5})$ the compounds $\mathbf{1 1}-\mathbf{1 3}$ feature approximately octahedral, 16 valence electron titanium centers, cis chloride ligands and approximately linear imido ligands. The main purposes of this crystallographic study were (i) to determine whether the structural consequences of varying the imido N-R substituent persisted between the two triazacyclic precatalyst systems $\operatorname{Ti}(\mathrm{NR})\left(\mathrm{Me}_{3}[n] \mathrm{aneN}_{3}\right) \mathrm{Cl}_{2}(n=6,9)$ and (ii) to evaluate the structural effects of changing the macrocycle ring size.

As for the $\mathrm{Me}_{3}[9] \mathrm{aneN}_{3}$ series, the tert-butyl imido ligand in $\mathbf{1 0}$ has the shortest $\operatorname{Ti}(1)-\mathrm{N}(1)$ bond length while the longest are found for $\mathbf{1 1}$ and 13. The trends in the $\mathrm{N}(1)-\mathrm{C}(10)$ distances are also analogous in the two series. The average $\mathrm{Ti}-\mathrm{Cl}$ distance in $\mathbf{1 0}$ is again longer than in the aryl imido homologs. All four compounds show the strong trans influence of the imido ligands, but there is little experimentally significant difference between their magnitudes as formally quantified by the differences between the $\operatorname{Ti}(1)-\mathrm{N}(6)$ and $\operatorname{Ti}(1)-\mathrm{N}(2)$ or $\operatorname{Ti}(1)-\mathrm{N}(4)$ distances. However, just as in the $\mathrm{Me}_{3}[9]$ aneN 3 series, the Ti-N distances $(\mathrm{Ti}(1)-\mathrm{N}(6))$ trans to the $\mathrm{NPh}(\mathbf{1 2})$ and $\mathrm{NC}_{6} \mathrm{~F}_{5}(\mathbf{1 3})$ ligands are significantly shorter than in the other two systems. Again only the perfluorophenyl imido analog (13) shows a pronounced supramolecular arrangement of the individual molecules. As shown in Fig. 4 this is also based on $\mathrm{C}(\delta+) \cdots \mathrm{F}(\delta$-) contacts (range 3.124(2) - 3.433(2) $\AA$ ) and the $\mathrm{C}_{6} \mathrm{~F}_{5}$ rings are once more significantly offset with regard to each other (Figs. S5 - S7). 
Several significant differences are associated with changing the macrocycle size. Most notably the average $\mathrm{N}_{\text {ring }}-\mathrm{Ti}-\mathrm{N}_{\text {ring }}$ angle in the $\mathrm{Me}_{3}[9]$ aneN $\mathrm{N}_{3}$ series $\mathbf{1}-\mathbf{5}$ is significantly larger than in $\mathbf{1 0}$ 13 (e.g. for 1 it is $75.6^{\circ}$ whereas in $\mathbf{1 0}$ it is $59.8^{\circ}$ ). Also noteworthy is the way that the macrocycle $\mathrm{N}$-methyl groups are oriented more away from metal centre in the $\mathrm{Me}_{3}[6] \mathrm{aneN}_{3}$ series (for example the average Ti-N-Me angle in $\mathbf{1}$ is $111.2^{\circ}$ whereas in $\mathbf{1 0}$ it is $130.5^{\circ}$ ). The average Ti-Cl and Ti$\mathrm{N}_{\text {ring }}$ distances in $\mathbf{1}-\mathbf{5}$ are both significantly longer than in $\mathbf{1 0}-\mathbf{1 3}$. The longer distances in the $\mathrm{Me}_{3}[9] \mathrm{aneN}_{3}$ series are attributed to the fact that the $\mathrm{N}$ donors of the macrocycle lie more strictly trans to the $\mathrm{Ti}-\mathrm{Cl}$ bonds due to the larger ring size. Similar trends have been found in the homologous pairs $(n=6$ or 9$) \mathrm{Sc}\left(\mathrm{Me}_{3}[n] \mathrm{aneN}_{3}\right) \mathrm{Cl}_{3}{ }^{10}$ and $\mathrm{Fe}\left(\mathrm{Me}_{3}[n] \mathrm{aneN}_{3}\right) \mathrm{Cl}_{3} \cdot{ }^{11,12}$ In contrast, the $\mathrm{Ti}=\mathrm{N}_{\text {imide }}$ distances for a given $\mathrm{N}-\mathrm{R}$ group are generally not significantly different between the two series (the exception being 5 and $\mathbf{1 3}\left(\Delta_{\mathrm{Ti}=\mathrm{N}}=0.012(1) \AA\right)$. However, the pronounced $\mathrm{C}_{6} \mathrm{~F}_{5} \cdots \mathrm{C}_{6} \mathrm{~F}_{5}$ supramolecular interactions could in principle influence the $\mathrm{Ti}=\mathrm{NC}_{6} \mathrm{~F}_{5}$ distances). 

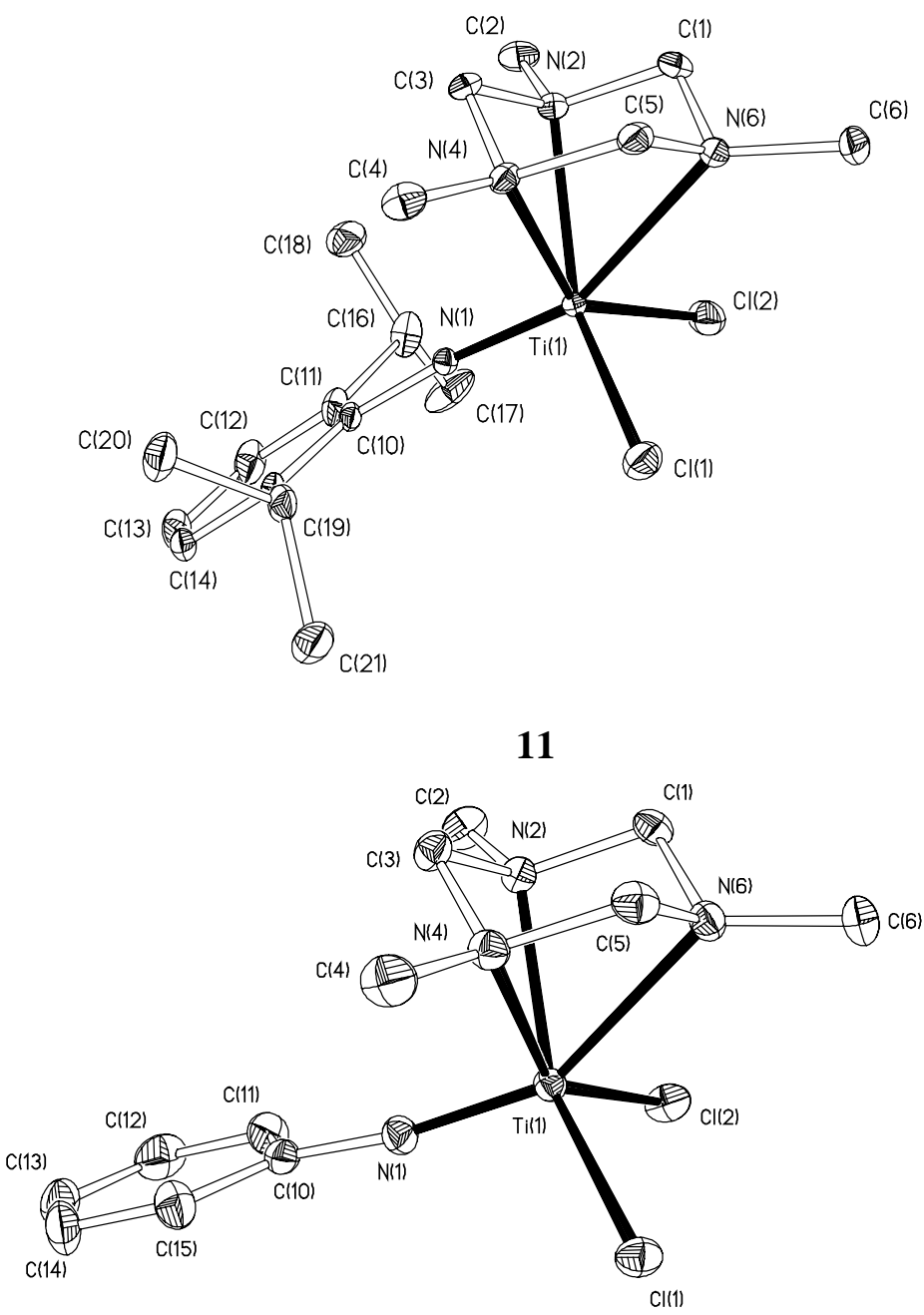

12



13

Figure S4. Displacement ellipsoid plots (20\% probability) of $\mathrm{Ti}(\mathrm{NR})\left(\mathrm{Me}_{3}[6] \mathrm{aneN}_{3}\right) \mathrm{Cl}_{2}(\mathrm{R}=2,6-$ $\mathrm{C}_{6} \mathrm{H}_{3}{ }^{\mathrm{i}} \mathrm{Pr}_{2}(\mathbf{1 1}), \mathrm{Ph}\left(\mathbf{1 2}\right.$, one of the crystallographically independent molecules) $\left.\mathrm{C}_{6} \mathrm{~F}_{5}(\mathbf{1 3})\right)$. $\mathrm{H}$ atoms omitted. 




Figure S5. Partial packing diagram for $\mathrm{Ti}\left(\mathrm{NC}_{6} \mathrm{~F}_{5}\right)\left(\mathrm{Me}_{3}[6] \mathrm{aneN}_{3}\right) \mathrm{Cl}_{2}(\mathbf{1 3})$. $\mathrm{H}$ atoms omitted and all atoms drawn as spheres of arbitrary radius. Symmetry operators: B, $[-x,-1-y,-z]$; C, $[-x,-y,-z]$. The dashed lines indicate some of the closest points of intermolecular contact: $\mathrm{C}(12) \cdots \mathrm{F}(3 \mathrm{C})=3.433(2)$ $\AA ; \mathrm{C}(13) \cdots \mathrm{F}(3 \mathrm{C})=3.344(2) \AA ; \mathrm{C}(14) \cdots \mathrm{F}(3 \mathrm{C})=3.422(2) \AA ; \mathrm{C}(13) \cdots \mathrm{C}(13 \mathrm{C})=3.224(4)$, $\mathrm{F}(4) \cdots \mathrm{C}(10 \mathrm{~B})=3.278(2) \AA ; \mathrm{F}(4) \cdots \mathrm{C}(15 \mathrm{~B})=3.124(2) \AA ; \mathrm{F}(5) \cdots \mathrm{C}(13 \mathrm{~B})=3.200(2) \AA ; \mathrm{F}(5) \cdots \mathrm{C}(14 \mathrm{~B})$ $=3.307(2) \AA$. 


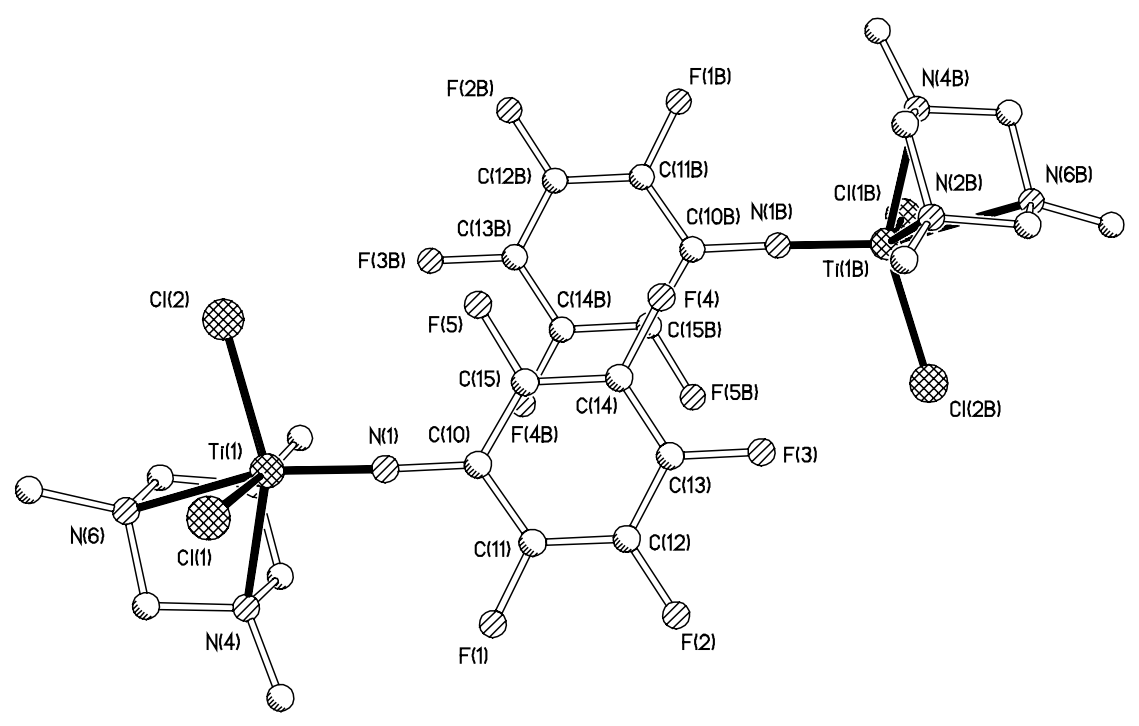

Figure S6. Relationship between the $\mathrm{C}_{6} \mathrm{~F}_{5}$ groups associated with $\operatorname{Ti}(1)$ and $\operatorname{Ti}(1 \mathrm{~B})$ in molecules of $\mathrm{Ti}\left(\mathrm{NC}_{6} \mathrm{~F}_{5}\right)\left(\mathrm{Me}_{3}[6] \mathrm{aneN}_{3}\right) \mathrm{Cl}_{2}(\mathbf{1 3})$. View is projected strictly onto the $\{\mathrm{C}(10), \mathrm{C}(11), \mathrm{C}(12), \mathrm{C}(13)$, $\mathrm{C}(14), \mathrm{C}(15)\}$ least-squares plane. H atoms omitted and all atoms drawn as spheres of arbitrary radius. Symmetry operator: $\mathrm{B},[-x,-1-y,-z]$

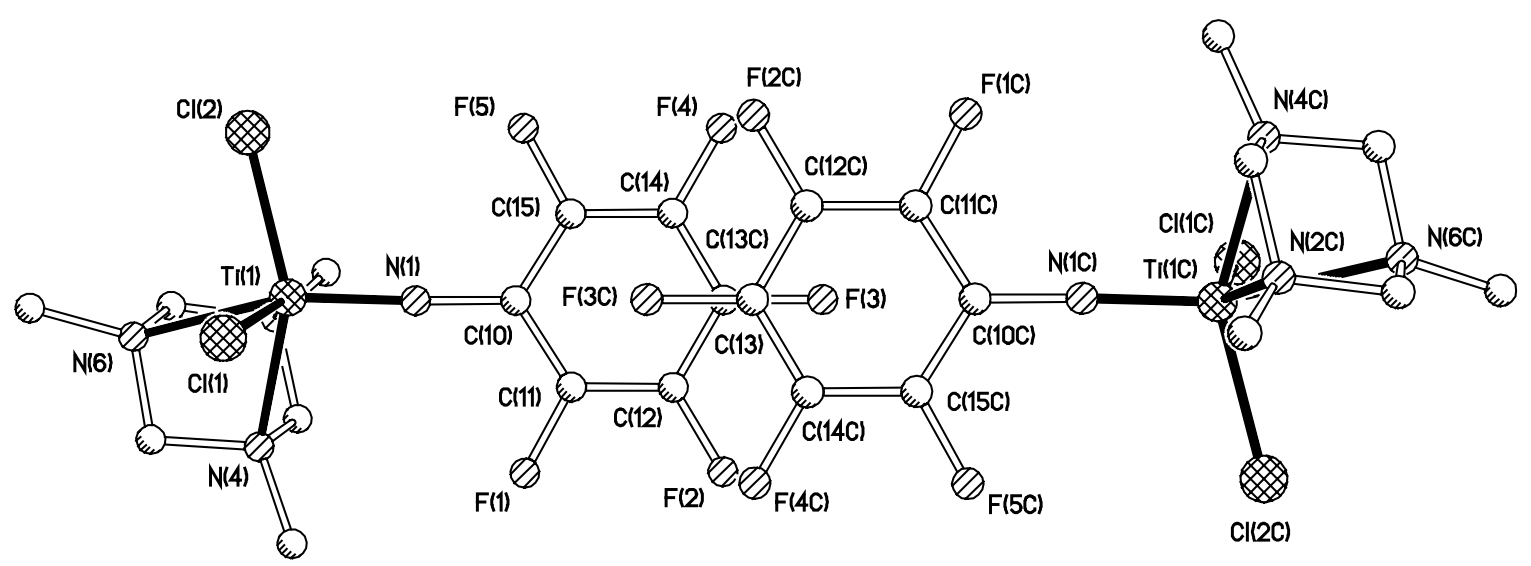

Figure S7. Relationship between the $\mathrm{C}_{6} \mathrm{~F}_{5}$ groups associated with $\mathrm{Ti}(1)$ and $\mathrm{Ti}(1 \mathrm{C})$ in molecules of $\mathrm{Ti}\left(\mathrm{NC}_{6} \mathrm{~F}_{5}\right)\left(\mathrm{Me}_{3}[6] \mathrm{aneN}_{3}\right) \mathrm{Cl}_{2}(\mathbf{1 3})$. View is projected strictly onto the $\{\mathrm{C}(10), \mathrm{C}(11), \mathrm{C}(12), \mathrm{C}(13)$, $\mathrm{C}(14), \mathrm{C}(15)\}$ least-squares plane. $\mathrm{H}$ atoms omitted and all atoms drawn as spheres of arbitrary radius. Symmetry operator: $\mathrm{C},[-x,-y,-z]$. 


\section{Experimental details for the synthesis of polystyrene-supported catalysts and $R_{3}[6] a^{2} N_{3}-$ supported catalysts.}

See main text experimental section for general details and procedures.

Starting materials. The compounds $\mathrm{Me}_{3}[6] \mathrm{aneN}_{3},{ }^{13} \operatorname{dd}_{3}[6] \mathrm{aneN}_{3},{ }^{13} \mathrm{LiMe}_{2}[9] \mathrm{aneN}_{3},{ }^{7}$ $\mathrm{Ti}\left(\mathrm{NCH}_{2} \mathrm{PS}\right) \mathrm{Cl}_{2}\left(\mathrm{NHMe}_{2}\right)_{2},{ }^{1}$ were prepared according to the literature methods. Chloromethyl polystyrene resin (Novabiochem, 100-200 mesh, loading $1.60 \mathrm{mmol}^{-1}{ }^{-1}$ ) was washed extensively with benzene, THF, dichloromethane and pentane (to remove any low molecular weight PS fractions) and dried under dynamic vacuum ( $1 \times 10^{-3}$ mbar, $\left.24 \mathrm{~h}\right)$ prior to use. .

$\mathbf{T i}\left(\mathbf{N C H}_{2} \mathbf{P S}\right)\left(\mathbf{M e}_{3}[\mathbf{9}] \mathbf{a n e N} \mathbf{N}_{\mathbf{3}}\right) \mathbf{C l}_{\mathbf{2}}$ (7). The dark red-brown resin $\mathrm{Ti}\left(\mathrm{NCH}_{2} \mathrm{PS}\right) \mathrm{Cl}_{2}\left(\mathrm{NHMe}_{2}\right)_{2}(0.45$ g, loading 1.19 mmol.g $\left.{ }^{-1}, 0.54 \mathrm{mmol}\right)$ was swollen in $\mathrm{C}_{6} \mathrm{H}_{6}(10 \mathrm{ml})$ for $30 \mathrm{~min}$. Subsequently $\mathrm{Me}_{3}[9] \mathrm{aneN}_{3}(0.14 \mathrm{ml}, 0.80 \mathrm{mmol}, 1.5$ equiv.) was added and the mixture shaken for $16 \mathrm{~h}$ during which time the dark red brown colour faded. Subsequently the resin was filtered off and washed with $\mathrm{C}_{6} \mathrm{H}_{6}(3 \times 10 \mathrm{ml})$ and $\mathrm{CH}_{2} \mathrm{Cl}_{2}(3 \times 10 \mathrm{ml})$, to give an pale cream material. Yield: $<95 \%$. ${ }^{1} \mathrm{H}$ MAS NMR (399.9 MHz, $4 \mathrm{kHz}$ spinning, swollen in $\mathrm{CDCl}_{3}$ ): 7.1 (v. br., polystyrene aromatic $\mathrm{H}$ ), 6.6 (v. br., polystyrene aromatic $\mathrm{H}$ ), 5.1 (br., $\mathrm{NC}_{2} \mathrm{Ph}$ ), 3.0 (v. br., $\mathrm{NMe}$ cis to $\mathrm{NCH}_{2} \mathrm{Ph}$ ), 2.4 (v. br., NMe trans to $\mathrm{NCH}_{2} \mathrm{Ph}$ ), 1.9 (v. br., polystyrene aliphatic $\mathrm{H}$ ), 1.4 (v. br. m, polystyrene aliphatic $\mathrm{H}$ ). ${ }^{13} \mathrm{C}-\left\{{ }^{1} \mathrm{H}\right\}$ MAS NMR (100.6 MHz, $4 \mathrm{kHz}$ spinning, swollen in $\mathrm{CDCl}_{3}$ ): 144.9 (br., polystyrene aromatic C), 127.6 (v. br., polystyrene aromatic C), 125.4 (v. br., polystyrene aromatic C), 72.9 $\left(\mathrm{NCH}_{2} \mathrm{Ph}\right), 45.8,43.5,40.3$ (3 x v. br., polystyrene aliphatic and other $\mathrm{C}$ resonances. IR $(\mathrm{KBr}$ pellet, $\mathrm{cm}^{-1}$ ): 3082 (s), 3000 (s), 2850 (s), 2818 (s), 2780 (s), 1944 (m), 1872 (m), 1802 (m), 1744 (m), 1708 (w), 1600 (s), 1582 (m), 1490 (s), 1442 (m), 1422 (m), 1364 (w), 1320 (w), 1292 (w), 1264 (w), 1232 (w), 1182 (w), 1152 (m), 1126 (w), 1060 (s), 1026 (m), 998 (m), 906 (m), 892 (m), $784(\mathrm{~m}), 738(\mathrm{~m}), 660(\mathrm{~m})$. Elemental analysis of the product repeatedly returned lower than expected $\% \mathrm{~N}, \mathrm{Cl}, \mathrm{Ti}$ values. This is attributed to incomplete combustion or acid digestion. The spectroscopic data and other experimental observations are consistent with quantitative uptake of $\mathrm{Me}_{3}[9] \mathrm{aneN}_{3}$ to form the desired 7.

$\mathbf{P S C H}_{2} \mathbf{M e}_{2}[9] \mathbf{a n e N}_{3}(\mathbf{8})$. To chloromethyl polystyrene (Merrifield resin) (0.52 g, loading 1.60 mmol.g $\left.{ }^{-1}, 0.83 \mathrm{mmol}\right)$, pre-swollen in THF $(20 \mathrm{ml})$ for $2 \mathrm{~h}$, was added a solution of $\mathrm{LiMe}_{2}[9] \mathrm{aneN}_{3}$ $(0.30 \mathrm{~g}, 1.84 \mathrm{mmol})$ in THF $(5 \mathrm{ml})$. Almost immediately after the addition, the resin changed colour from off-white to deep burgundy. The mixture was shaken for $16 \mathrm{~h}$ and the resin subsequently filtered off. It was washed with THF ( $5 \times 20 \mathrm{ml})$, EtOH ( $3 \times 5 \mathrm{ml}$ ) (red colour quenched) and $\mathrm{Et}_{2} \mathrm{O}$ $(3 \times 10 \mathrm{ml})$ and dried for $16 \mathrm{~h}$ under reduced pressure to give a very pale yellow resin. Yield: $94 \%$. Elemental analysis indicated a loading of $1.51 \mathrm{mmol} . \mathrm{g}^{-1} \quad{ }^{1} \mathrm{H}$ MAS NMR $(399.9 \mathrm{MHz}, 4 \mathrm{kHz}$, swollen in $\mathrm{CDCl}_{3}$ ): 7.1 (br., polystyrene aromatic $\mathrm{H}$ ), 6.5 (br., polystyrene aromatic $\mathrm{H}$ ), 3.6 (br., 
$\mathrm{NC}_{2} \mathrm{PS}$ ), 2.8 (br. m, $\mathrm{CH}_{2}$ ring), 2.65 (overlapping 2 x br. m, 2 x $\mathrm{CH}_{2}$ ring), 2.35 (br., $\mathrm{NMe),} 1.9$ 1.4 (br. polystyrene aliphatic H). ${ }^{13} \mathrm{C}-\left\{{ }^{1} \mathrm{H}\right\}$ MAS NMR $\left(100.6 \mathrm{MHz}, 4 \mathrm{kHz}\right.$, swollen in $\left.\mathrm{CDCl}_{3}\right)$ : 145.1, 137.1 ( 2 x sharp, polystyrene aromatic C). 127.7, 125.5 ( 2 x br., polystyrene aromatic C), 62.9 (sharp, $\mathrm{NCH}_{2} \mathrm{PS}$ ), 56.9 (sharp, $\mathrm{CH}_{2}$ ring), 55.8 (sharp, 2 x overlapping, $2 \mathrm{x} \mathrm{CH}_{2}$ ring), 46.6 (sharp, NMe), 44.1, 40.2 (2 x br., polystyrene aliphatic C). IR (KBr pellet, $\mathrm{cm}^{-1}$ ): 3100 (s), 2668 (s), 1942 (m), 1868 (m), 1800 (m), 1742 (m), 1600 (s), 1582 (m), 1510 (m), 1490 (s), 1434 (s), 1416 (s), 1350 (s), 1264 (m), 1200 (m), 1178 (m), 1150 (m), 1092 (s), 1024 (s), 964 (m), 942 (m), 904 (m), $872(\mathrm{~m}), 822$ (m), 744 (m), 700 (m), 664 (m), 618 (w), 512 (m), 464 (m). Anal. found: C, 12.99; H, $7.75 ; \mathrm{Cl}, 0 ; \mathrm{N}, 6.36 \%$.

$\left.\mathbf{T i}\left(\mathbf{N}^{\mathrm{t}} \mathbf{B u}\right)\left(\mathbf{P S C H}_{2} \mathbf{M e}_{2}[\mathbf{9}] \mathbf{a n e N}\right)_{3}\right) \mathbf{C l}_{2}$ (9). $\operatorname{PSCH}_{2} \mathrm{Me}_{2}[9] \operatorname{aneN}_{3}(\mathbf{8}, 0.30 \mathrm{~g}$, macrocycle loading: $\left.1.51 \mathrm{mmol} \mathrm{g}^{-1}, 0.45 \mathrm{mmol}\right)$ was swollen in benzene $(15 \mathrm{ml})$ for $30 \mathrm{~min}$. Subsequently a solution of $\operatorname{Ti}\left(\mathrm{N}^{\mathrm{t} B u}\right) \mathrm{Cl}_{2}\left(\mathrm{NHMe}_{2}\right)_{2}(0.20 \mathrm{~g}, 0.72 \mathrm{mmol}, 1.6$ equivs. $)$ in benzene $(5 \mathrm{ml})$ was added and the mixture shaken for $16 \mathrm{~h}$. Soon after addition of the imido complex, the solution discoloured. The resin was filtered off, washed with benzene $(3 \times 10 \mathrm{ml})$, THF $(5 \times 10 \mathrm{ml})$, toluene $(3 \times 10 \mathrm{ml})$ and $\mathrm{CH}_{2} \mathrm{Cl}_{2}(3 \mathrm{x} 10 \mathrm{ml})$ and dried in vacuo for $16 \mathrm{~h}$. Yield quantitative. ${ }^{1} \mathrm{H}$ MAS NMR $(399.9 \mathrm{MHz}, 4$ $\mathrm{kHz}$, swollen in $\mathrm{CD}_{2} \mathrm{Cl}_{2}$ ): 7.2 (v. br., polystyrene aromatic $\mathrm{H}$ ), 6.7 (v. br., polystyrene aromatic $\mathrm{H}$ ), 5.3 (br., $\mathrm{NCH}_{2} \mathrm{PS}$ ), 3.4 (v. br., $\mathrm{NMe}$ cis to $\mathrm{N}^{\mathrm{t}} \mathrm{Bu}$ ), 3.2 (v. br., $\mathrm{CH}_{2}$ ring), 2.9 (v. br., $\mathrm{CH}_{2}$ ring), 2.6 (v. br., NMe trans to $\mathrm{N}^{\mathrm{t} B u}$ ), 1.9, 1.6 (v. br., polystyrene resin backbone), 1.3 (br., $\left.{ }^{\mathrm{t}} \mathrm{Bu}\right) .{ }^{13} \mathrm{C}-\left\{{ }^{1} \mathrm{H}\right\}$ MAS NMR (100.6 MHz, $4 \mathrm{kHz}$, swollen in $\mathrm{CD}_{2} \mathrm{Cl}_{2}$ ): 166.6, 145.6 (2 x br., polystyrene aromatic C), 131.9 (br., $\mathrm{NCH}_{2} \underline{\mathrm{Ph}}(\mathrm{PS})$ ), 128.2, 125.9 ( 2 x v. br., polystyrene aromatic C), 69.1 (sharp, $\underline{\mathrm{CH}}_{2} \mathrm{NPS}$ ), 56.9 - 50.9 (series of v. br. signals, $\mathrm{CH}_{2}$ ring), 48.2 (sharp, $\mathrm{NMe}$ ), 44.1, 42.9 (2 x v. br., polystyrene aliphatic C), 40.5 (br., polystyrene aliphatic C), 31.1 (br., $\mathrm{NCMe}_{3}$ ). IR (KBr pellet, $\mathrm{cm}^{-}$ 1): 3082 (s), 3056 (s), 3029 (s), 2854 (s), 2820 (s), 1944 (w), 1870 (w), 1802 (w), 1600 (m), 1582 (m), 1510 (m), 1492 (s), 1452 (s), 1420 (m), 1378 (w), 1350 (m), 1328 (w), 1294 (w), 1240 (s), 1206 (m), 1182 (m), 1146 (m), 1082 (m), 1066 (m), 1024 (m), 1004 (m), 990 (m), 966 (m), 948 (m), $900(\mathrm{~m}), 814(\mathrm{~m}), 748(\mathrm{~m}), 736(\mathrm{~m}), 696(\mathrm{~m}), 646$ (w), $518(\mathrm{~m}), 492(\mathrm{~m})$. Elemental analysis of the product repeatedly returned lower than expected $\% \mathrm{~N}, \mathrm{Cl}, \mathrm{Ti}$ values.

$\mathbf{T i}(\mathbf{N P h})\left(\mathbf{M e}_{3}[\mathbf{6}] \mathbf{a n e N} \mathbf{N}_{\mathbf{3}}\right) \mathbf{C l}_{\mathbf{2}}(\mathbf{1 2})$. To a stirred solution of $\mathrm{Ti}\left(\mathrm{NMe}_{2}\right)_{2} \mathrm{Cl}_{2}(0.398 \mathrm{~g}, 1.92 \mathrm{mmol})$ in benzene $(20 \mathrm{ml})$ was added $\mathrm{PhNH}_{2}(0.175 \mathrm{ml}, 1.92 \mathrm{mmol})$. The initially red solution darkened immediately before gradually brightening to red. After $2 \mathrm{~h}, \mathrm{Me}_{3}[6] \mathrm{aneN}_{3}(0.28 \mathrm{ml}, 2 \mathrm{mmol})$ was added to afford a pink precipitate, and after a further $2 \mathrm{~h}$ the mixture was filtered and the precipitate dried under reduced pressure to yield a yellow powder which was washed with hexanes $(2 \times 5 \mathrm{ml})$ and dried in vacuo. Yield: $0.450 \mathrm{~g}(69 \%)$. ${ }^{1} \mathrm{H} \mathrm{NMR}\left(300.2 \mathrm{MHz}, \mathrm{CD}_{2} \mathrm{Cl}_{2}, 257 \mathrm{~K}\right): 7.04$ (2 H, app. t, app. ${ }^{3} \mathrm{~J}=8.2 \mathrm{~Hz}$, meta- $\left.\mathrm{C}_{6} \mathrm{H}_{5}\right), 6.82-6.71\left(3 \mathrm{H}\right.$, overlapping $\mathrm{m}$, ortho- and para- $\left.\mathrm{C}_{6} \mathrm{H}_{5}\right), 4.70(1 \mathrm{H}, \mathrm{d}$, $\left.{ }^{2} \mathrm{~J}=8.1 \mathrm{~Hz}, \mathrm{CH}_{2} \operatorname{ring}\right), 4.22\left(2 \mathrm{H}, \mathrm{d},{ }^{2} \mathrm{~J}=8.7 \mathrm{~Hz}, \mathrm{CH}_{2} \operatorname{ring}\right), 3.86\left(1 \mathrm{H}, \mathrm{d},{ }^{2} \mathrm{~J}=8.4 \mathrm{~Hz}, \mathrm{CH}_{2} \operatorname{ring}\right)$, 
$3.53\left(1 \mathrm{H}, \mathrm{d},{ }^{2} \mathrm{~J}=8.7 \mathrm{~Hz}, \mathrm{CH}_{2}\right.$ ring), $2.77(6 \mathrm{H}, \mathrm{s}, \mathrm{NMe}$ cis to $\mathrm{NPh}), 2.23(3 \mathrm{H}, \mathrm{s}, \mathrm{NMe}$ trans to $\mathrm{NPh})$. ${ }^{13} \mathrm{C}-\left\{{ }^{1} \mathrm{H}\right\}$ NMR $\left(75.5 \mathrm{MHz}, \mathrm{CD}_{2} \mathrm{Cl}_{2}, 238 \mathrm{~K}\right.$ ): 159.1 (ipso- $\mathrm{C}_{6} \mathrm{H}_{5}$ ), 128.1 (meta- $\mathrm{C}_{6} \mathrm{H}_{5}$ ), 122.8 (para$\mathrm{C}_{6} \mathrm{H}_{5}$ ), 121.5 (ortho- $\left.\mathrm{C}_{6} \mathrm{H}_{5}\right), 77.8\left(\mathrm{CH}_{2}\right.$ ring), $76.6\left(\mathrm{CH}_{2}\right.$ ring), 41.2 (NMe trans to $\left.\mathrm{NPh}\right), 37.3(\mathrm{NMe}$ trans to NPh). IR (KBr, cm $\left.{ }^{-1}\right): 3062$ (m), 2990 (m), 2932 (m), 2832 (m), 1576 (m), 1474 (s), 1452 (m), 1432 (w), 1382 (w), 1322 (s), 1268 (m), 1174 (w), 1116 (s), 1080 (m), 1066 (m), 970 (m), 932 (s), $776(\mathrm{~s}), 696(\mathrm{~m}), 512(\mathrm{~m}), 490(\mathrm{w}) . \quad$ EI-MS: $m / z=338(9 \%)[\mathrm{M}]^{+}, 208(10 \%)[\mathrm{M}-$ $\left.\mathrm{Me}_{3}[6] a n e \mathrm{~N}_{3}\right]^{+}$. Anal. found (calculated for $\mathrm{C}_{12} \mathrm{H}_{20} \mathrm{Cl}_{2} \mathrm{~N}_{4} \mathrm{Ti}$ ): C, 42.5 (42.2); H, 5.9 (6.3); N, 16.5 (16.6) \%.

$\mathbf{T i}\left(\mathbf{N C}_{\mathbf{6}} \mathbf{F}_{5}\right)\left(\mathbf{M e}_{3}[\mathbf{6}] \mathbf{a n e N} \mathbf{N}_{\mathbf{3}}\right) \mathbf{C l}_{2}$ (13). To a stirred solution of $\mathrm{Ti}\left(\mathrm{NMe}_{2}\right)_{2} \mathrm{Cl}_{2}(1.208 \mathrm{~g}, 5.84$ mmol) in benzene $(20 \mathrm{ml})$ was added a solution of $\mathrm{C}_{6} \mathrm{~F}_{5} \mathrm{NH}_{2}(1.07 \mathrm{~g}, 5.84 \mathrm{mmol})$ in benzene (20 $\mathrm{ml}$ ). The initially red solution darkened immediately before gradually brightening back to red. After $2 \mathrm{~h}, \mathrm{Me}_{3}[6] \mathrm{aneN}_{3}(0.90 \mathrm{ml}, 6.42 \mathrm{mmol})$ was added to afford an orange precipitate, and after a further $2 \mathrm{~h}$ the mixture was filtered and the precipitate dried under reduced pressure to yield an a pale orange powder which was washed with hexanes $(2 \mathrm{x} 10 \mathrm{ml})$ and dried in vacuo. Yield: $2.32 \mathrm{~g}$ (93\%). ${ }^{1} \mathrm{H}$ NMR (300.2 MHz, $\left.\mathrm{CD}_{2} \mathrm{Cl}_{2}, 243 \mathrm{~K}\right): 4.80\left(1 \mathrm{H}, \mathrm{d},{ }^{2} \mathrm{~J}=8.1 \mathrm{~Hz}, \mathrm{CH}_{2} \operatorname{ring}\right), 4.32\left(2 \mathrm{H}, \mathrm{d},{ }^{2} \mathrm{~J}\right.$ $\left.=8.7 \mathrm{~Hz}, \mathrm{CH}_{2} \operatorname{ring}\right), 3.90\left(2 \mathrm{H}, \mathrm{d},{ }^{2} \mathrm{~J}=8.1 \mathrm{~Hz}, \mathrm{CH}_{2} \operatorname{ring}\right), 3.62\left(2 \mathrm{H}, \mathrm{d},{ }^{2} \mathrm{~J}=8.7 \mathrm{~Hz}, \mathrm{CH}_{2}\right.$ ring $), 2.77$ (6 H, s, NMe cis to $\left.\mathrm{NC}_{6} \mathrm{~F}_{5}\right), 2.33$ (3 H, s, NMe trans to $\left.\mathrm{NC}_{6} \mathrm{~F}_{5}\right) .{ }^{19} \mathrm{~F}$ NMR $\left(282.4 \mathrm{MHz}, \mathrm{CD}_{2} \mathrm{Cl}_{2}\right.$, $243 \mathrm{~K}):-154.9\left(2 \mathrm{~F}, \mathrm{~d},{ }^{3} \mathrm{~J}=18.3 \mathrm{~Hz}\right.$, ortho- $\left.\mathrm{C}_{6} \mathrm{~F}_{5}\right),-166.9\left(2 \mathrm{~F}, \mathrm{dd},{ }^{3} \mathrm{~J}=18.3 \mathrm{~Hz}\right.$, meta- $\left.\mathrm{C}_{6} \mathrm{~F}_{5}\right),-168.5$ $\left(1 \mathrm{~F}, \mathrm{t},{ }^{3} \mathrm{~J}=21.4 \mathrm{~Hz}\right.$, para $\left.-\mathrm{C}_{6} \mathrm{~F}_{5}\right) .{ }^{13} \mathrm{C}-\left\{{ }^{1} \mathrm{H}\right\} \mathrm{NMR}\left(75.5 \mathrm{MHz}, \mathrm{CD}_{2} \mathrm{Cl}_{2}, 243 \mathrm{~K}\right): 78.0\left(\mathrm{CH}_{2}\right.$ ring), $76.7\left(\mathrm{CH}_{2}\right.$ ring), $41.3\left(\mathrm{NMe}\right.$ cis to $\left.\mathrm{NC}_{6} \mathrm{~F}_{5}\right), 37.3$, ( $\mathrm{NMe}$ trans to $\mathrm{NC}_{6} \mathrm{~F}_{5}$ ), the $\mathrm{C}_{6} \mathrm{~F}_{5}$ ring carbons were not observed due to ${ }^{19} \mathrm{~F}$ coupling. IR ( $\mathrm{NaCl}$ plates, Nujol, $\mathrm{cm}^{-1}$ ): 1510 (s), 1490 (s), 1480 (s), 1464 (s), 1450 (s), 1266 (w), 1222 (m), 1122 (w) 1110 (m), 1056 (s), 1006 (w), 980(s), 936 (m), 416 (w). EI-MS: $m / z=428(43 \%)[\mathrm{M}]^{+}, 299(19 \%)\left[\mathrm{M}-\mathrm{Me}_{3}[6] \mathrm{aneN}_{3}\right]^{+}$. Anal. found (calculated for $\mathrm{C}_{12} \mathrm{H}_{15} \mathrm{Cl}_{2} \mathrm{~F}_{5} \mathrm{~N}_{4} \mathrm{Ti} \cdot 0.1\left(\mathrm{CH}_{2} \mathrm{Cl}_{2}\right.$ ), sample crystallized from $\left.\mathrm{CH}_{2} \mathrm{Cl}_{2}\right)$ : C, 33.1 (33.3); H, 4.0 (3.5); $\mathrm{N}$, $13.2(12.9) \%$.

$\mathbf{T i}\left(\mathbf{N}^{t} \mathbf{B u}\right)\left(\mathbf{d d}_{3}[\mathbf{6}] \mathbf{a n e N} \mathbf{N}_{\mathbf{3}}\right) \mathbf{C l}_{\mathbf{2}}(\mathbf{1 4})$. To a solution of $\mathrm{Ti}\left(\mathrm{N}^{\mathrm{t} B u}\right) \mathrm{Cl}_{2}\left(\mathrm{HNMe}_{2}\right)_{2}(0.215 \mathrm{~g}, 0.77 \mathrm{mmol})$ in pentane $(20 \mathrm{ml})$ was added $\mathrm{dd}_{3}[6] \operatorname{aneN}_{3}(0.412 \mathrm{~g}, 0.70 \mathrm{mmol})$ also dissolved in pentane $(20 \mathrm{ml})$. The resulting yellow solution was stirred for $2 \mathrm{~h}$, filtered at $0{ }^{\circ} \mathrm{C}$, and the volatiles were removed and the yellow wax (which could not be crystallized) dried in vacuo. Yield: $0.407 \mathrm{~g},(75 \%) .{ }^{1} \mathrm{H}$ NMR (500.0 MHz, $\left.\mathrm{CD}_{2} \mathrm{Cl}_{2}\right): 4.80\left(1 \mathrm{H}, \mathrm{d},{ }^{2} \mathrm{~J}=7.5 \mathrm{~Hz}, \mathrm{CH}_{2} \operatorname{ring}\right), 4.16\left(2 \mathrm{H}, \mathrm{d},{ }^{2} \mathrm{~J}=8.0 \mathrm{~Hz}, \mathrm{CH}_{2}\right.$ ring), $3.73\left(1 \mathrm{H}, \mathrm{d},{ }^{2} \mathrm{~J}=7.5 \mathrm{~Hz}, \mathrm{CH}_{2} \operatorname{ring}\right), 3.45\left(2 \mathrm{H}, \mathrm{d},{ }^{2} \mathrm{~J}=8.0 \mathrm{~Hz}, \mathrm{CH}_{2} \operatorname{ring}\right), 3.10(4 \mathrm{H}, \mathrm{m}$, $\mathrm{NCH}_{2}\left(\mathrm{CH}_{2}\right)_{10} \mathrm{Me}$ cis to $\left.\mathrm{N}^{t} \mathrm{Bu}\right), 2.39\left(2 \mathrm{H}, \mathrm{t},{ }^{3} \mathrm{~J}=8.5 \mathrm{~Hz}, \mathrm{CH}_{2}\left(\mathrm{CH}_{2}\right){ }_{10} \mathrm{Me}\right.$ trans to $\left.\mathrm{N}^{\mathrm{t}} \mathrm{Bu}\right), 1.73(4 \mathrm{H}$. $\mathrm{m}, \mathrm{NCH}_{2} \underline{\mathrm{C}}_{2}\left(\mathrm{CH}_{2}\right)_{9} \mathrm{Me}$ cis to $\mathrm{N}^{\mathrm{t}} \mathrm{Bu}$ ), 1.37 - 1.09 (56 H, series of overlapping $\mathrm{m}, \mathrm{C}_{2}$ dodecyl chain), 1.00, $\left(9 \mathrm{H}, \mathrm{s},{ }^{\mathrm{t}} \mathrm{Bu}\right), 0.85\left(9 \mathrm{H}, \mathrm{m}, \mathrm{N}\left(\mathrm{CH}_{2}\right)_{11} \underline{\mathrm{Me}}\right) .{ }^{13} \mathrm{C}-\left\{{ }^{1} \mathrm{H}\right\} \mathrm{NMR}\left(125.7 \mathrm{MHz}, \mathrm{CD}_{2} \mathrm{Cl}_{2}\right): 75.1$ $\left(\mathrm{CH}_{2}\right.$ ring), $74.2 \quad\left(\mathrm{CH}_{2}\right.$ ring), $70.4 \quad\left(\mathrm{CMe}_{3}\right), \quad 55.9 \quad\left(\mathrm{NCH}_{2}\left(\mathrm{CH}_{2}\right)_{10} \mathrm{Me}\right.$ cis to $\left.\mathrm{N}^{t} \mathrm{Bu}\right), \quad 52.0$ 
$\left(\mathrm{NCH}_{2}\left(\mathrm{CH}_{2}\right)_{10} \mathrm{Me}\right.$ trans to $\left.\mathrm{N}^{\mathrm{t} B u}\right), 32.3\left(\mathrm{NCH}_{2}\left(\mathrm{CH}_{2}\right)_{9} \underline{\mathrm{CH}_{2}} \mathrm{Me}\right), 31.4\left(\mathrm{CMe}_{3}\right), 30.0$ - 29.7 (series of overlapping signals, $\quad \underline{\mathrm{CH}}_{2}$ dodecyl chain), $27.7 \quad\left(\mathrm{NCH}_{2} \mathrm{CH}_{2} \underline{\mathrm{CH}}_{2}\left(\mathrm{CH}_{2}\right)_{9} \mathrm{Me}\right), \quad 25.3$ $\left(\mathrm{NCH}_{2} \underline{\mathrm{CH}_{2}}\left(\mathrm{CH}_{2}\right)_{9} \mathrm{Me}\right.$ cis to $\left.\mathrm{N}^{t} \mathrm{Bu}\right), 24.8 \quad\left(\mathrm{NCH}_{2} \mathrm{CH}_{2}\left(\mathrm{CH}_{2}\right)_{9} \mathrm{Me}\right.$ trans to $\left.\mathrm{N}^{t} \mathrm{Bu}\right), 23.1$ $\left(\mathrm{NCH}_{2}\left(\mathrm{CH}_{2}\right)_{8} \underline{\mathrm{CH}}_{2} \mathrm{CH}_{2} \mathrm{Me}\right) 14.3\left(\mathrm{~N}\left(\mathrm{CH}_{2}\right)_{11} \underline{\mathrm{Me}}\right)$. IR ( $\mathrm{NaCl}$ plates, Nujol, $\left.\mathrm{cm}^{-1}\right)$ : 2850 (s, br), 1464 (s, br), 1378 (m), 1350 (m), 1332 (m), 1244 (m, br), 1096 (m br), 1018 (m) 938 (m), 896 (w), 802 (m), 722(m), 594 (w). Anal. found (calculated for $\mathrm{C}_{43} \mathrm{H}_{90} \mathrm{Cl}_{2} \mathrm{~N}_{4} \mathrm{Ti}$ ): C, 65.6 (66.0); H, 12.0 (11.6); N 7.0 (7.2) $\%$.

Ti(N-2,6-C $\left.\mathbf{C}_{\mathbf{6}} \mathbf{H}_{\mathbf{3}}{ }^{\mathrm{i}} \mathbf{P r}_{\mathbf{2}}\right)\left(\mathbf{d d}_{\mathbf{3}}[\mathbf{6}] \mathbf{a n n e N}_{\mathbf{3}}\right) \mathbf{C l}_{\mathbf{2}}$ (15). To a stirred solution of $\mathrm{Ti}\left(\mathrm{NMe}_{2}\right)_{2} \mathrm{Cl}_{2}(0.225 \mathrm{~g}, 1.09$ $\mathrm{mmol})$ in benzene $(10 \mathrm{ml})$ was added 2,6-diisopropylaniline $(0.21 \mathrm{ml}, 1.09 \mathrm{mmol})$. The initially red solution darkened immediately before gradually brightening to red again. After $18 \mathrm{~h}$, a solution of $\mathrm{dd}_{3}[6] \operatorname{aneN}_{3}(0.65 \mathrm{~g}, 1.09 \mathrm{mmol})$ in benzene $(20 \mathrm{ml})$ was added. The resulting red solution was stirred for $3 \mathrm{~h}$ before the volatiles were removed under reduced pressure to afford a brown semisolid. This was dissolved in pentane $(20 \mathrm{ml})$ before being filtered at $0{ }^{\circ} \mathrm{C}$, and the volatiles were removed and the brown wax (which could not be crystallized) dried in vacuo. Yield: $0.764 \mathrm{~g}$, (79\%). ${ }^{1} \mathrm{H}$ NMR (500.0 MHz, $\left.\mathrm{CD}_{2} \mathrm{Cl}_{2}, 273 \mathrm{~K}\right): 6.86\left(2 \mathrm{H}, \mathrm{d},{ }^{3} \mathrm{~J}=7.5 \mathrm{~Hz}\right.$, meta $\left.-\mathrm{C}_{6} \underline{\mathrm{H}}_{3}{ }^{\mathrm{i}} \mathrm{Pr}_{2}\right), 6.70(1$ $\mathrm{H}, \mathrm{d},{ }^{3} \mathrm{~J}=7.5 \mathrm{~Hz}$, para- $\left.\mathrm{C}_{6} \underline{\mathrm{H}}_{3}{ }^{\mathrm{i}} \mathrm{Pr}_{2}\right), 4.61\left(1 \mathrm{H}, \mathrm{d},{ }^{2} \mathrm{~J}=8.0 \mathrm{~Hz}, \mathrm{CH}_{2} \operatorname{ring}\right), 4.37\left(2 \mathrm{H}, \mathrm{d},{ }^{2} \mathrm{~J}=7.5 \mathrm{~Hz}\right.$, $\mathrm{CH}_{2}$ ring), 4.26 (2 H, sept., $\left.{ }^{3} \mathrm{~J}=7.0 \mathrm{~Hz}, \mathrm{CHMe}_{2}\right), 4.00\left(1 \mathrm{H}, \mathrm{d},{ }^{2} \mathrm{~J}=8.0 \mathrm{~Hz}, \mathrm{CH}_{2} \operatorname{ring}\right), 3.66(2 \mathrm{H}, \mathrm{d}$, ${ }^{2} \mathrm{~J}=8.0 \mathrm{~Hz}, \mathrm{CH}_{2}$ ring), $3.30\left(2 \mathrm{H}, \mathrm{dt},{ }^{2} \mathrm{~J}=13.5 \mathrm{~Hz},{ }^{3} \mathrm{~J}=5.0 \mathrm{~Hz}, \mathrm{NCH}_{2}\left(\mathrm{CH}_{2}\right)_{10} \mathrm{Me}\right.$ cis to NAr), 3.09 $\left(2 \mathrm{H}, \mathrm{dt},{ }^{2} \mathrm{~J}=13.5 \mathrm{~Hz},{ }^{3} \mathrm{~J}=5.0 \mathrm{~Hz}, \mathrm{NCH}_{2}\left(\mathrm{CH}_{2}\right)_{10} \mathrm{Me}\right.$ cis to $\left.\mathrm{NAr}\right), 2.60\left(2 \mathrm{H}\right.$, app. t, app. ${ }^{3} \mathrm{~J}=8.5 \mathrm{~Hz}$, $\mathrm{NC}_{2}\left(\mathrm{CH}_{2}\right)_{10} \mathrm{Me}$ trans to $\left.\mathrm{NAr}\right), 1.62\left(2 \mathrm{H}, \mathrm{m}, \mathrm{NCH}_{2} \underline{\mathrm{C}}_{2}\left(\mathrm{CH}_{2}\right)_{9} \mathrm{Me}\right.$ cis to $\left.\mathrm{NAr}\right), 1.50$ (2 $\mathrm{H}, \mathrm{m}$, $\mathrm{NCH}_{2} \mathrm{CH}_{2}\left(\mathrm{CH}_{2}\right)_{9} \mathrm{Me}$ cis to NAr), 1.37 (2 $\mathrm{H}, \mathrm{m}, \mathrm{NCH}_{2} \mathrm{CH}_{2}\left(\mathrm{CH}_{2}\right)_{9} \mathrm{Me}$ trans to NAr), 1.34 - 1.07 (54 $\mathrm{H}$, series of overlapping signals, $\mathrm{C}_{2} 2$ dodecyl chain), $1.19\left(12 \mathrm{H}, \mathrm{d},{ }^{3} \mathrm{~J}=6.5 \mathrm{~Hz}, \mathrm{CHMe} 2\right), 0.85(9$ $\left.\mathrm{H}, \mathrm{m}, \mathrm{N}\left(\mathrm{CH}_{2}\right){ }_{11} \mathrm{Me}\right) .{ }^{13} \mathrm{C}-\left\{{ }^{1} \mathrm{H}\right\}$ NMR $\left(125.7 \mathrm{MHz}, \mathrm{CD}_{2} \mathrm{Cl}_{2}, 273 \mathrm{~K}\right.$ ): 159.2 (ipso- $\mathrm{C}_{6} \mathrm{H}_{3}{ }^{\mathrm{i}} \mathrm{Pr}_{2}$ ), 133.7 (ortho- $\underline{\mathrm{C}}_{6} \mathrm{H}_{3}{ }^{\mathrm{i}} \mathrm{Pr}_{2}$ ), 127.3 (meta- $\underline{\mathrm{C}}_{6} \mathrm{H}_{3}{ }^{\mathrm{i}} \mathrm{Pr}_{2}$ ), 121.1 (para- $\underline{\mathrm{C}}_{6} \mathrm{H}_{3}{ }^{\mathrm{i}} \mathrm{Pr}_{2}$ ), $74.7\left(\mathrm{CH}_{2}\right.$ ring), $74.4\left(\mathrm{CH}_{2}\right.$ ring), $56.2\left(\mathrm{NCH}_{2}\left(\mathrm{CH}_{2}\right)_{10} \mathrm{Me}\right.$ cis to $\left.\mathrm{NAr}\right), \quad 52.1 \quad\left(\mathrm{NCH}_{2}\left(\mathrm{CH}_{2}\right)_{10} \mathrm{Me}\right.$ trans to $\left.\mathrm{NAr}\right), 32.2$

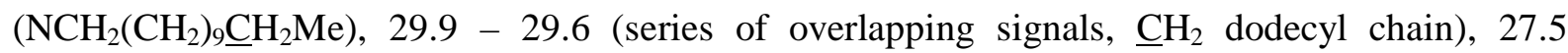
$(\underline{\mathrm{CHMe}} 2), 27.4\left(\mathrm{NCH}_{2} \mathrm{CH}_{2} \underline{\mathrm{CH}_{2}}\left(\mathrm{CH}_{2}\right)_{9} \mathrm{Me}\right), 24.3\left(\mathrm{NCH}_{2} \underline{\mathrm{CH}_{2}}\left(\mathrm{CH}_{2}\right)_{9} \mathrm{Me}\right.$ cis to $\left.\mathrm{NAr}\right), 24.1\left(\mathrm{CHMe}_{2}\right)$, $24.0\left(\mathrm{NCH}_{2} \mathrm{CH}_{2}\left(\mathrm{CH}_{2}\right)_{9} \mathrm{Me}\right.$ trans to NAr), $23.0\left(\mathrm{NCH}_{2}\left(\mathrm{CH}_{2}\right)_{8} \mathrm{CH}_{2} \mathrm{CH}_{2} \mathrm{Me}\right), 14.2\left(\mathrm{~N}\left(\mathrm{CH}_{2}\right)_{11} \underline{\mathrm{Me}}\right)$. IR ( NaCl plates, Nujol, $\mathrm{cm}^{-1}$ ): 2922 (s, br), 2850 (s, br), 1468 (m), 1424 (m), 1408 (m), 1378 (m), 1358 (w), 1328 (m), 1278 (m), 1114 (m), 1100 (m), 1044 (w), 974 (w), 942 (m), 804 (m), 762 (s), 722 (w). Anal. found (calculated for $\mathrm{C}_{51} \mathrm{H}_{98} \mathrm{Cl}_{2} \mathrm{~N}_{4} \mathrm{Ti}$ ): C, 68.5 (69.1); H, 11.2 (11.2); N 6.3 (6.3).

$\left.\mathbf{T i}\left(\mathbf{N}-2-\mathbf{C}_{6} \mathbf{H}_{4}{ }^{\mathrm{t}} \mathbf{B u}\right)\left(\mathbf{M e}_{3}[\mathbf{6}] \mathbf{a n e N}\right)_{3}\right) \mathbf{C l}_{2}$ (59). To a stirred solution of $\mathrm{Ti}\left(\mathrm{NMe}_{2}\right)_{2} \mathrm{Cl}_{2}(0.32 \mathrm{~g}, 1.54$ $\mathrm{mmol})$ in benzene $(20 \mathrm{ml})$ was added 2-tert-butyl aniline $(0.24 \mathrm{ml}, 1.54 \mathrm{mmol})$. The initially red solution darkened immediately before gradually brightening to orange. After $2 \mathrm{~h}, \mathrm{Me}_{3}[6] \mathrm{aneN}_{3}$ $(0.22 \mathrm{ml}, 1.54 \mathrm{mmol})$ was added to afford a red precipitate. After a further $2 \mathrm{~h}$ the mixture was 
filtered and the precipitate dried under reduced pressure to yield a pale orange powder. This was washed with pentane $(2 \times 15 \mathrm{ml})$ and dried in vacuo. Yield: $0.53 \mathrm{~g}(88 \%)$. ${ }^{1} \mathrm{H}$ NMR $(300.2 \mathrm{MHz}$, $\left.\mathrm{CD}_{2} \mathrm{Cl}_{2}, 273 \mathrm{~K}\right): 7.20\left(1 \mathrm{H}, \mathrm{dd},{ }^{3} \mathrm{~J}=8.1 \mathrm{~Hz},{ }^{4} \mathrm{~J}=1.5 \mathrm{~Hz}, 6-\mathrm{C}_{6} \underline{\mathrm{H}}_{4}{ }^{\mathrm{t}} \mathrm{Bu}\right), 7.05\left(1 \mathrm{H}, \mathrm{dd},{ }^{3} \mathrm{~J}=8.1 \mathrm{~Hz},{ }^{4} \mathrm{~J}=\right.$ $\left.1.5 \mathrm{~Hz}, 3-\mathrm{C}_{6} \underline{\mathrm{H}}_{4}{ }^{\mathrm{t}} \mathrm{Bu}\right), 6.94\left(1 \mathrm{H} . \mathrm{td},{ }^{3} \mathrm{~J}=7.5 \mathrm{~Hz},{ }^{4} \mathrm{~J}=1.5 \mathrm{~Hz}, 5-\mathrm{C}_{6} \underline{\mathrm{H}}_{4}{ }^{\mathrm{H}} \mathrm{Bu}\right), 6.67\left(1 \mathrm{H}, \mathrm{td},{ }^{3} \mathrm{~J}=8.1 \mathrm{~Hz},{ }^{4} \mathrm{~J}\right.$ $\left.=1.5 \mathrm{~Hz}, 4-\mathrm{C}_{6} \underline{\mathrm{H}}_{4}{ }^{\mathrm{t}} \mathrm{Bu}\right), 4.68\left(1 \mathrm{H}, \mathrm{d},{ }^{2} \mathrm{~J}=7.8 \mathrm{~Hz}, \mathrm{CH}_{2} \operatorname{ring}\right), 4.27\left(1 \mathrm{H}, \mathrm{d},{ }^{2} \mathrm{~J}=7.5 \mathrm{~Hz}, \mathrm{CH}_{2} \operatorname{ring}\right)$, $3.78\left(1 \mathrm{H}, \mathrm{d},{ }^{2} \mathrm{~J}=8.4 \mathrm{~Hz}, \mathrm{CH}_{2}\right.$ ring $), 3.44\left(2 \mathrm{H}, \mathrm{d},{ }^{2} \mathrm{~J}=9.0 \mathrm{~Hz}, \mathrm{CH}_{2}\right.$ ring $), 2.80(6 \mathrm{H}, \mathrm{s}, \mathrm{NMe}$ cis to NAr), 2.23 (3 H, s, NMe trans to NAr), $1.54\left(9 \mathrm{H}, \mathrm{s},{ }^{\mathrm{t}} \mathrm{Bu}\right) .{ }^{13} \mathrm{C}-\left\{{ }^{1} \mathrm{H}\right\} \mathrm{NMR}\left(75.5 \mathrm{MHz}, \mathrm{CD}_{2} \mathrm{Cl}_{2}, 273\right.$ $\mathrm{K}): 159.1\left(1-\underline{\mathrm{C}}_{6} \mathrm{H}_{4}{ }^{\mathrm{t}} \mathrm{Bu}\right), 140.3\left(2-\underline{\mathrm{C}}_{6} \mathrm{H}_{4}{ }^{\mathrm{t}} \mathrm{Bu}\right), 130.8\left(6-\underline{\mathrm{C}}_{6} \mathrm{H}_{4}{ }^{\mathrm{t}} \mathrm{Bu}\right), 125.9\left(5-\underline{\mathrm{C}}_{6} \mathrm{H}_{4}{ }^{\mathrm{t}} \mathrm{Bu}\right), 125.3$ (3$\left.\underline{\mathrm{C}}_{6} \mathrm{H}_{4}{ }^{\mathrm{B}} \mathrm{Bu}\right), 121.5\left(4-\underline{\mathrm{C}}_{6} \mathrm{H}_{4}{ }^{\mathrm{t}} \mathrm{Bu}\right), 78.0\left(\mathrm{CH}_{2}\right.$ ring), $76.8\left(\mathrm{CH}_{2}\right.$ ring), 41.0 (NMe cis to NAr), 37.3 (NMe trans to NAr), 35.3 ( $\left.\underline{\mathrm{CMe}}_{3}\right), 30.1\left(\mathrm{CMe}_{3}\right)$. IR (KBr, cm $\left.{ }^{-1}\right): 2958$ (br, s), 1482 (w), 1470 (s), 1452 (m), 1428 (m), 1384 (w), 1292 (m), 1268 (s), 1176 (w), 1122 (s), 1108 (m), 1082 (w), 1006 (w), $968(\mathrm{w}), 934$ (s), 774 (s). EI-MS: $m / z=394(10 \%)[\mathrm{M}]^{+}, 299(70 \%)\left[\mathrm{M}-\mathrm{Me}_{3}[6] \mathrm{aneN}_{3}\right]^{+}$. Found (calculated for $\mathrm{C}_{16} \mathrm{H}_{28} \mathrm{Cl}_{2} \mathrm{~N}_{4} \mathrm{Ti}$ ): C, 48.7 (48.6); $\mathrm{H}, 6.8$ (7.1); N, 14.2 (14.2). 


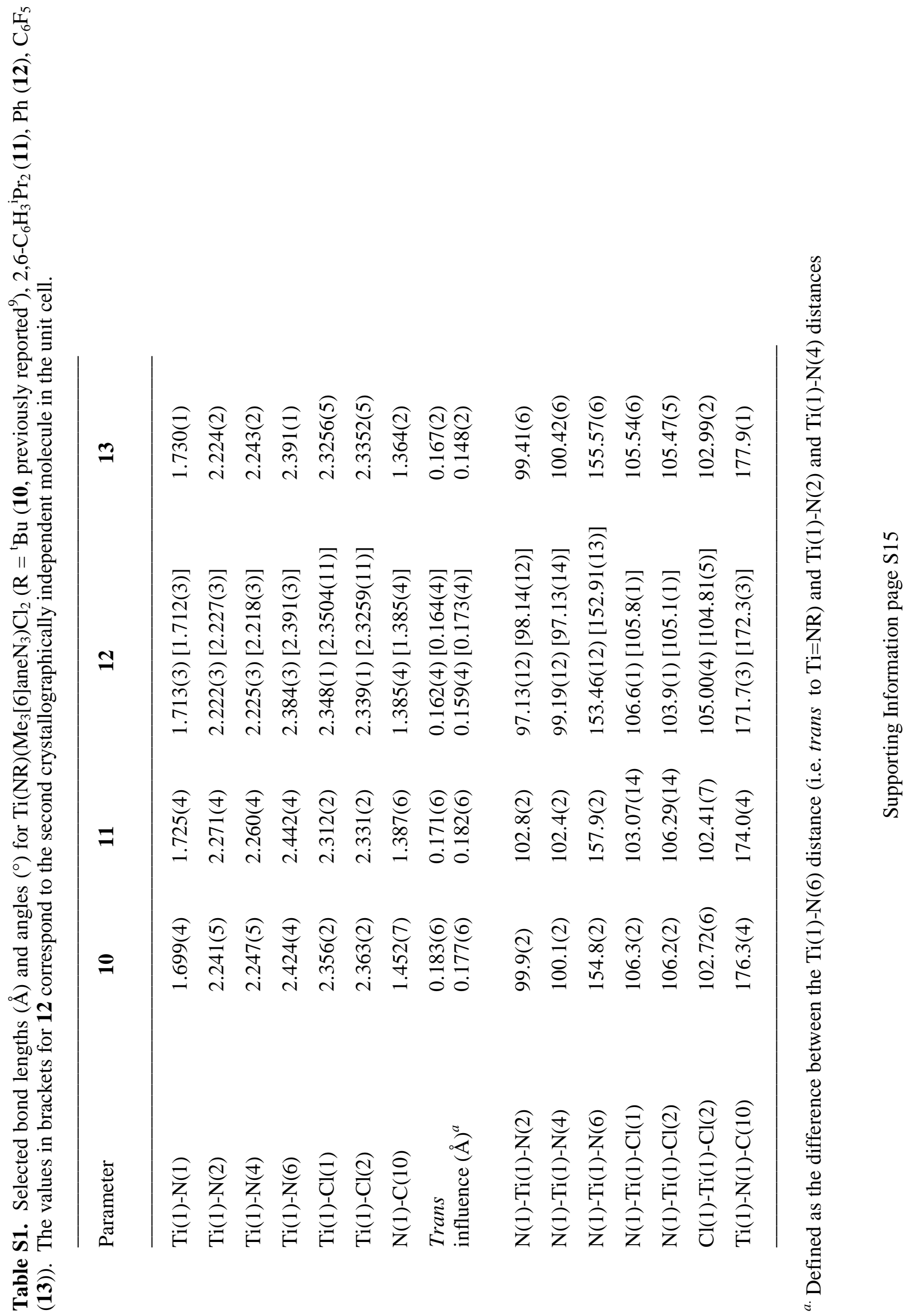




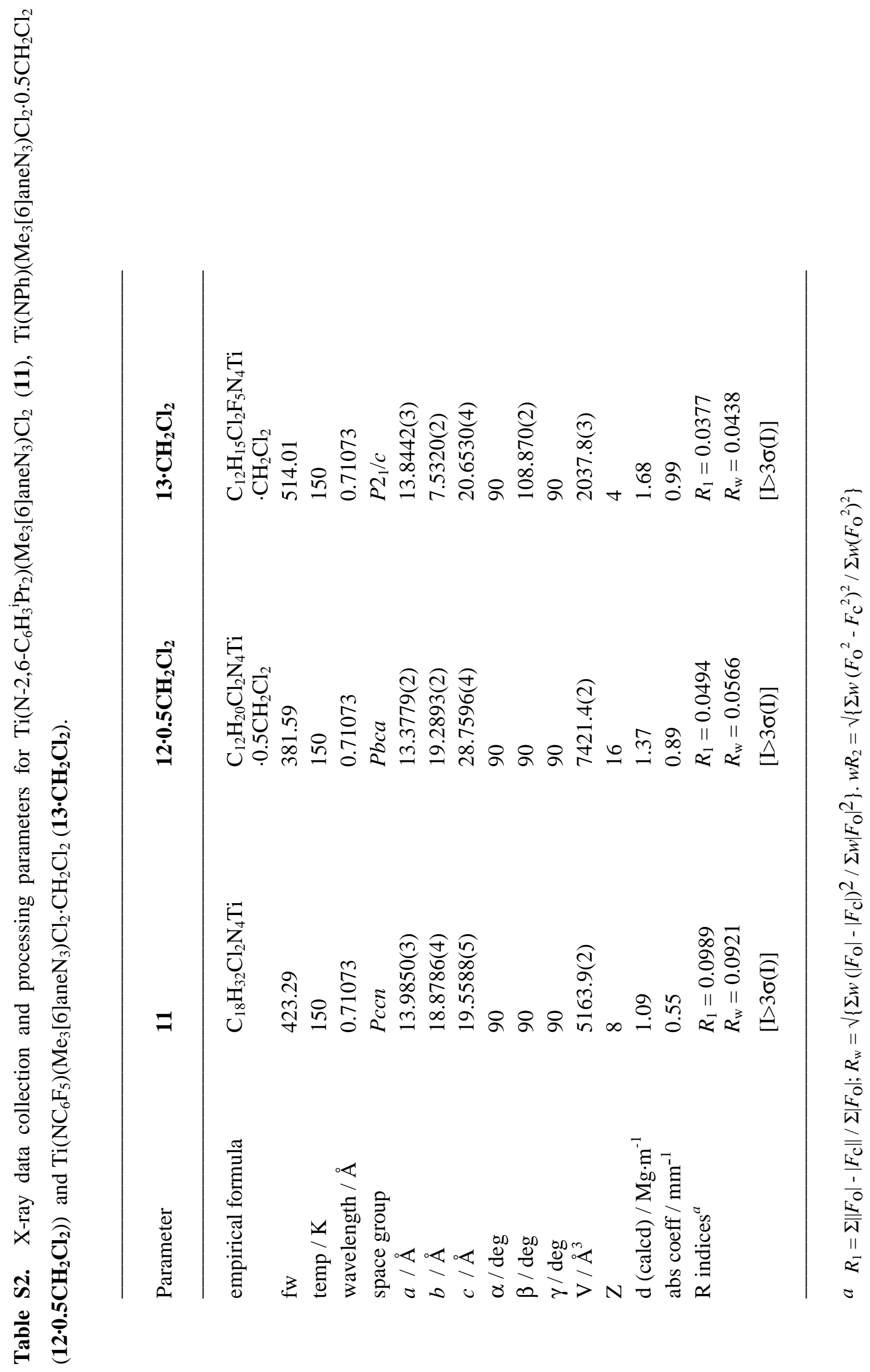




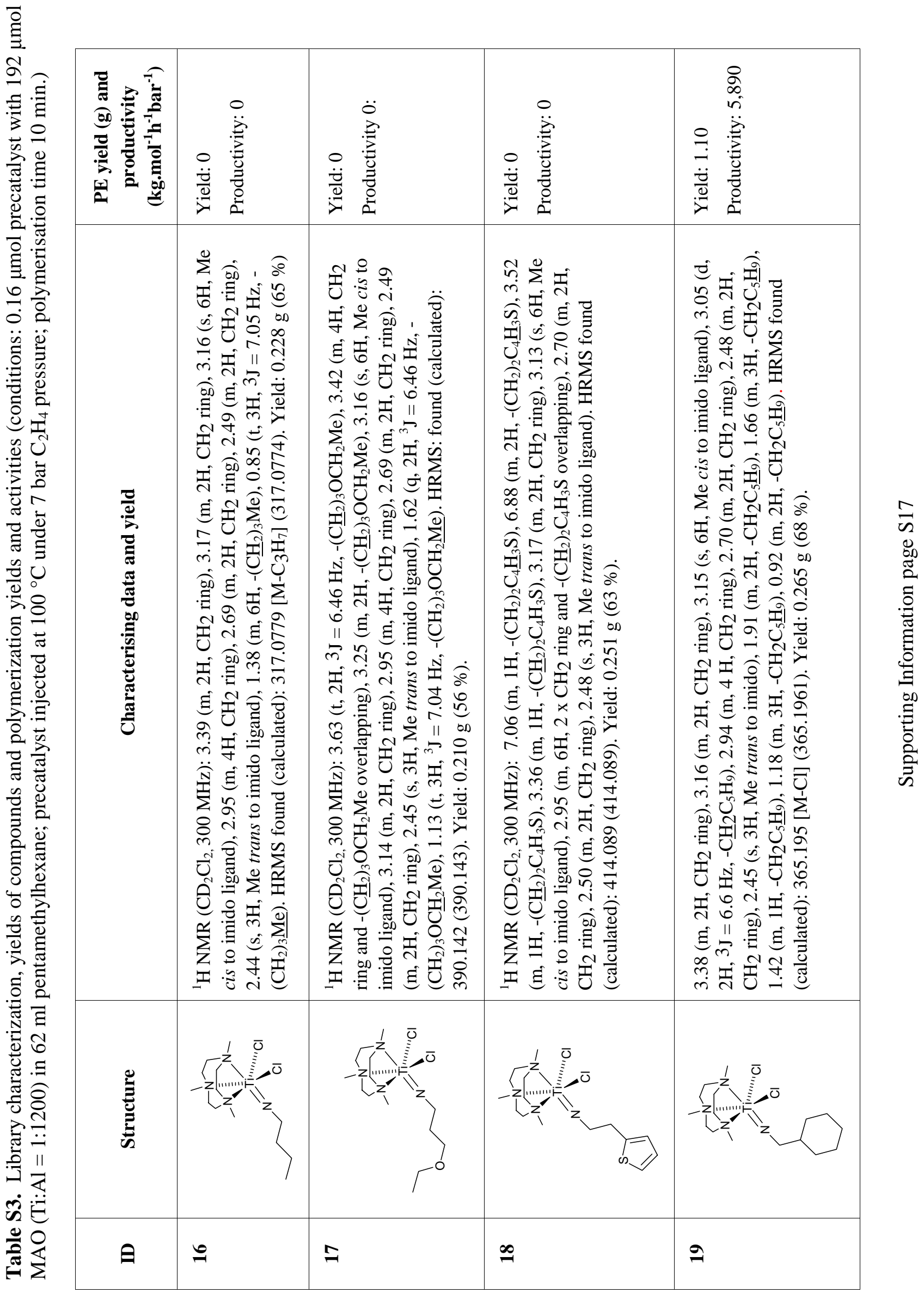




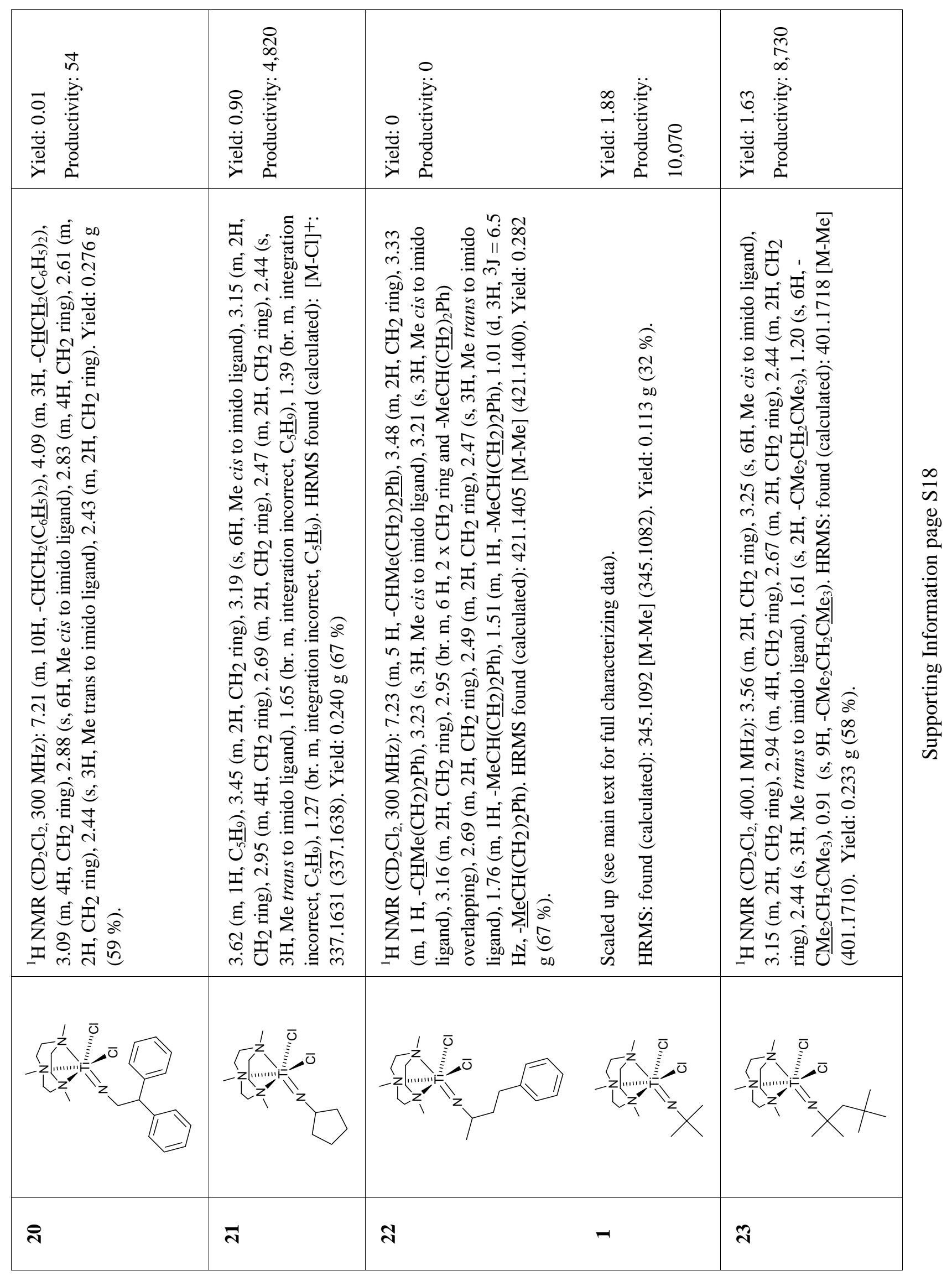




\begin{tabular}{|c|c|c|c|}
\hline 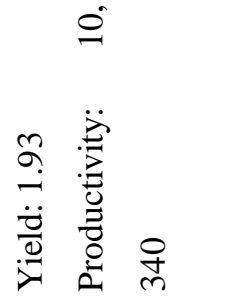 & 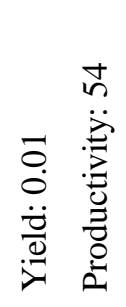 & 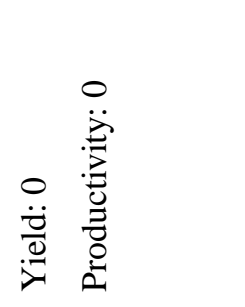 & 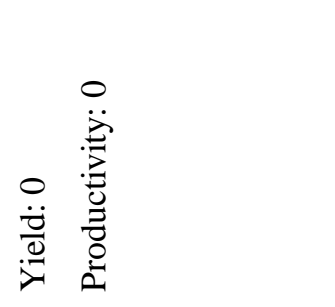 \\
\hline 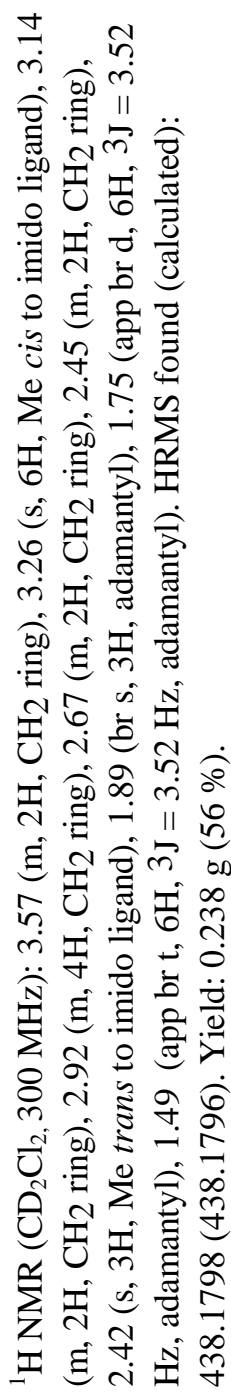 & 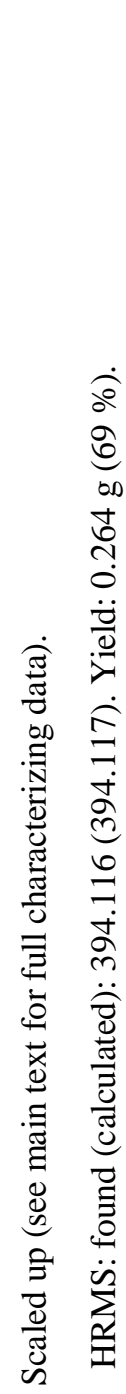 & 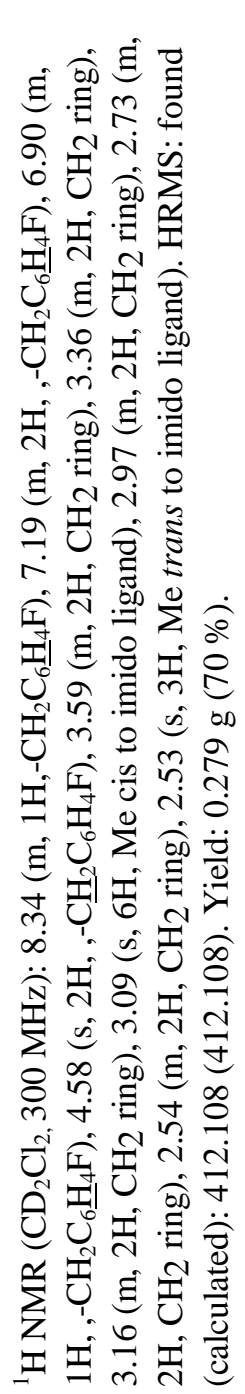 & 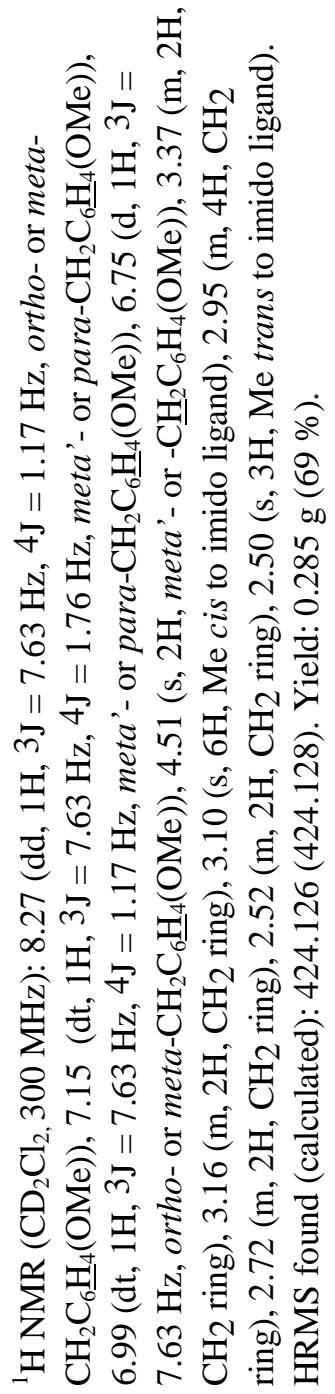 \\
\hline & & & \\
\hline$\sqrt[4]{ }$ & $b$ & $\mathbb{N}$ & $\sim$ \\
\hline
\end{tabular}

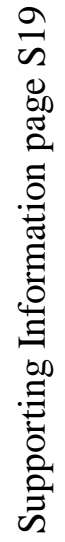




\begin{tabular}{|c|c|c|c|}
\hline 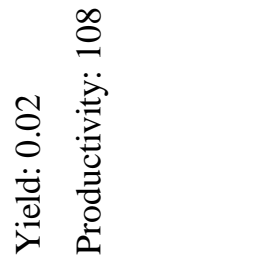 & 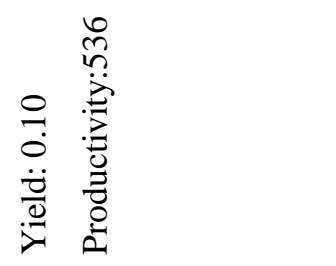 & 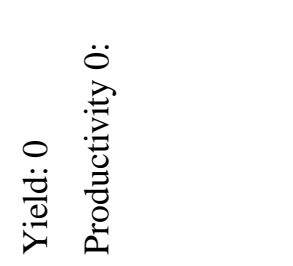 & 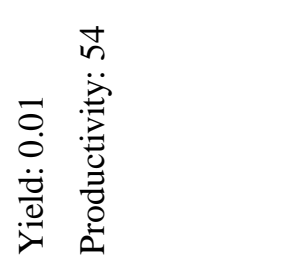 \\
\hline 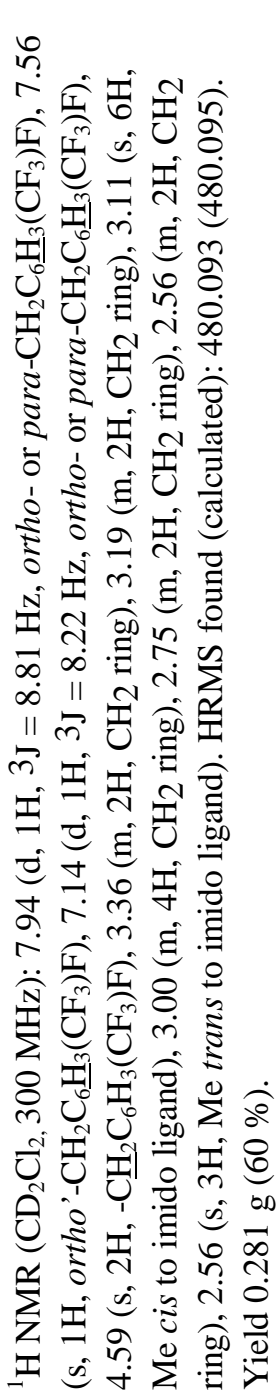 & 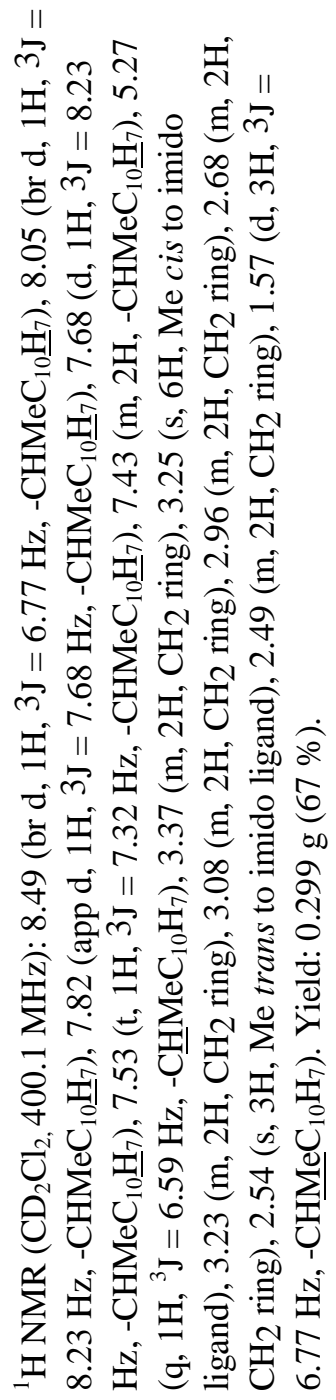 & 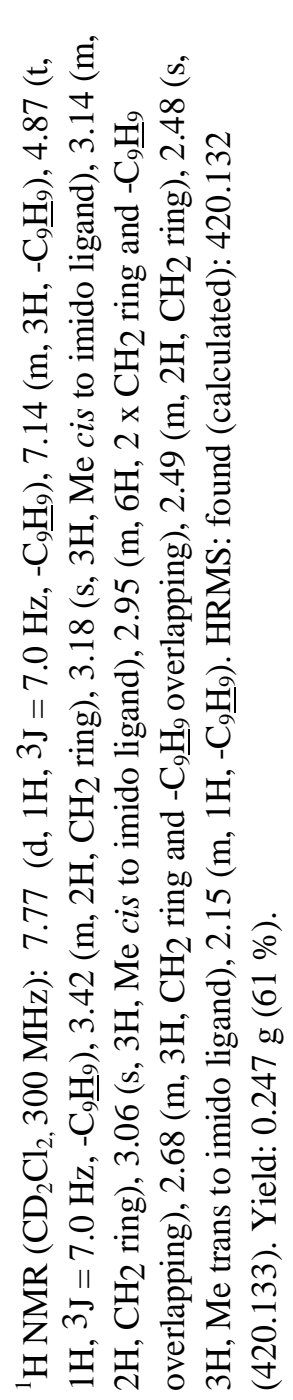 & 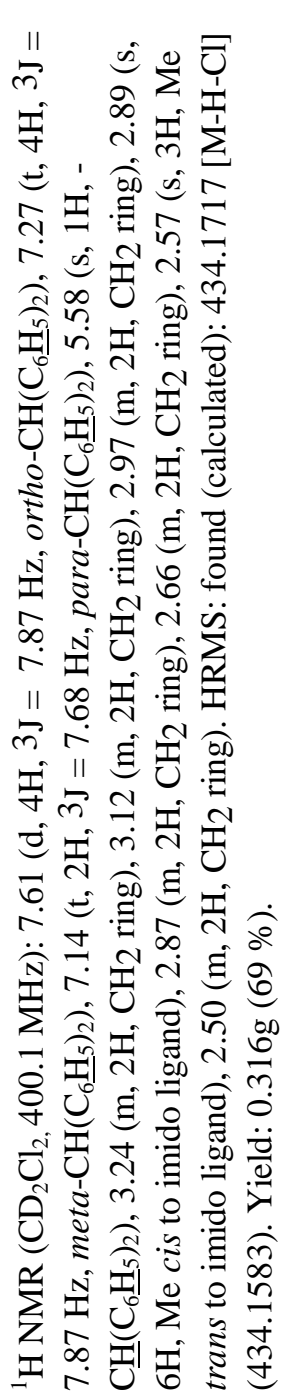 \\
\hline & & & \\
\hline$\grave{ง}$ & 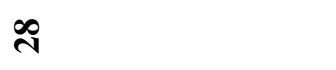 & ते & m \\
\hline
\end{tabular}




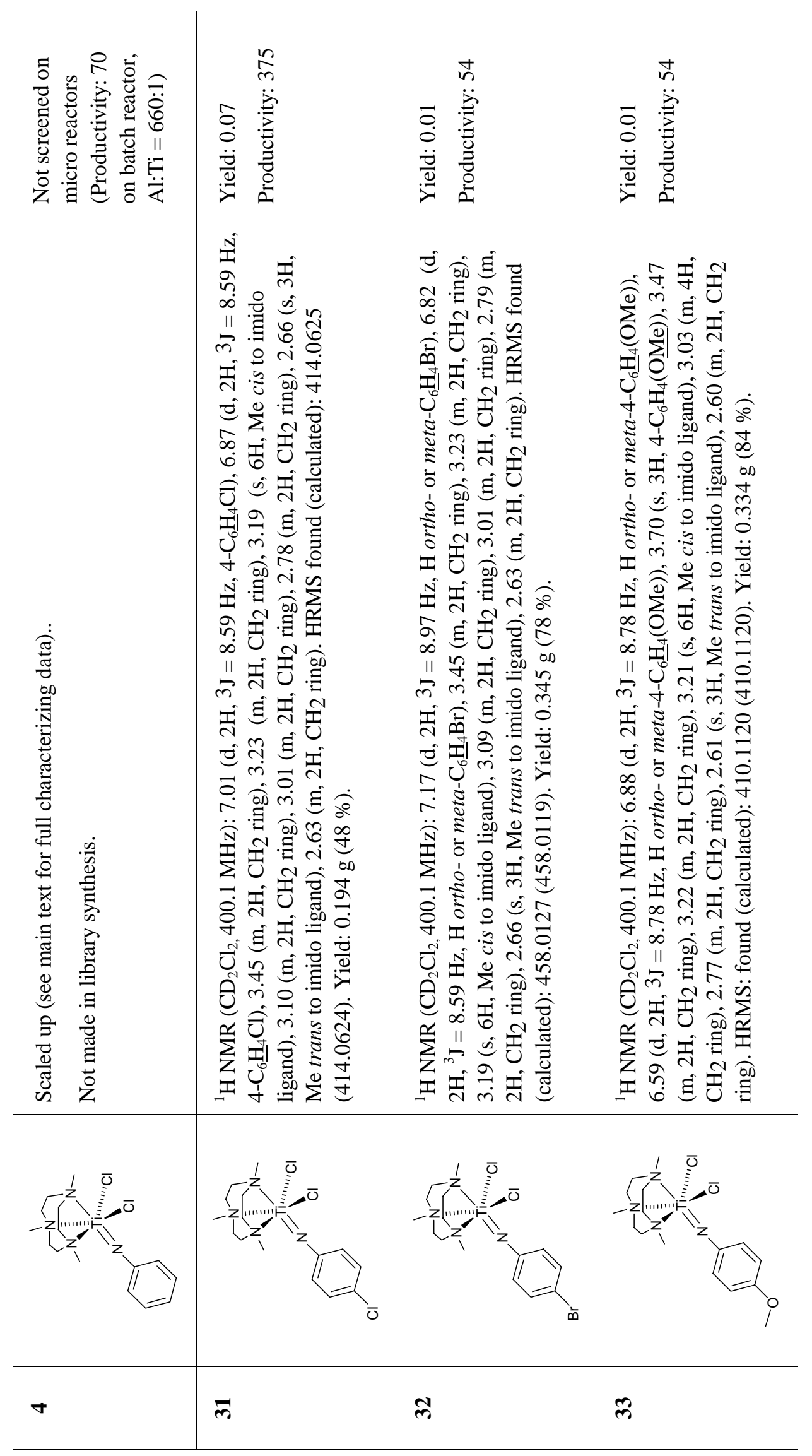

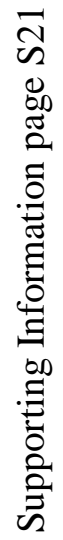




\begin{tabular}{|c|c|c|c|}
\hline 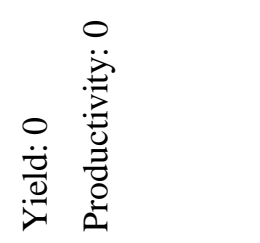 & 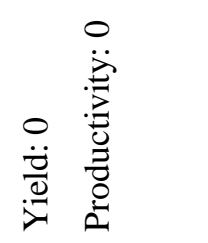 & 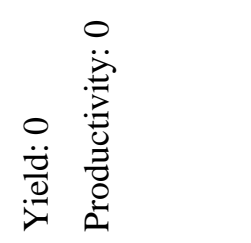 & 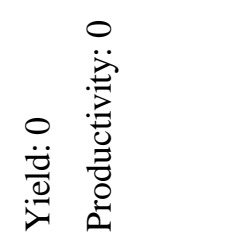 \\
\hline 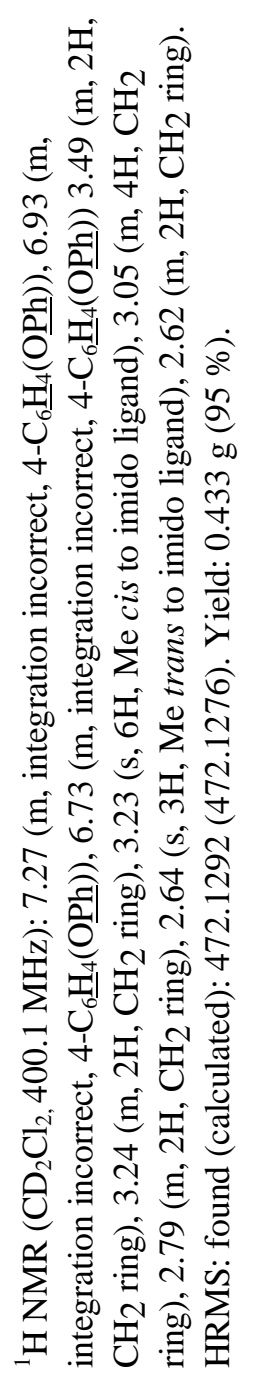 & 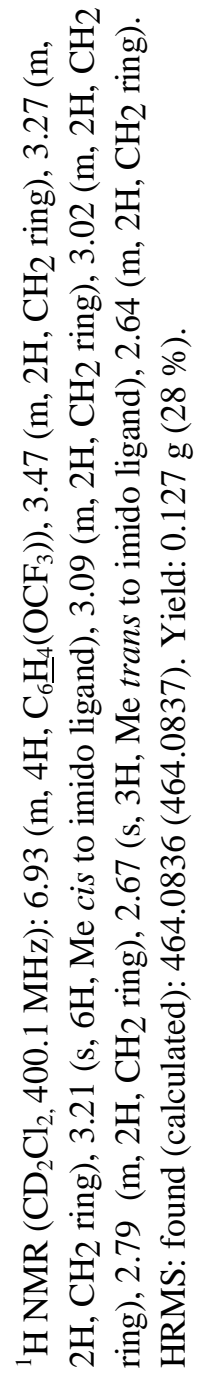 & 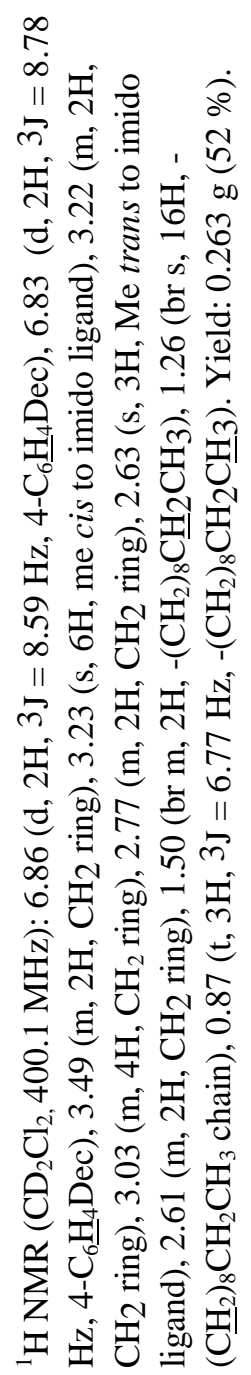 & 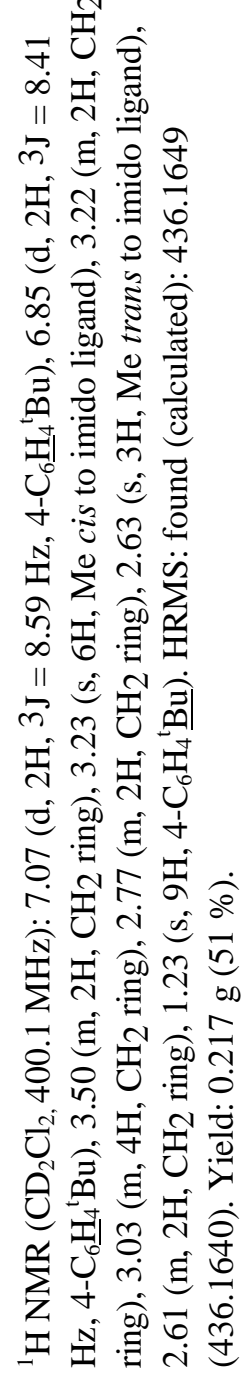 \\
\hline & & & \\
\hline ले & $\ddot{m}$ & ri & m \\
\hline
\end{tabular}




\begin{tabular}{|c|c|c|c|}
\hline 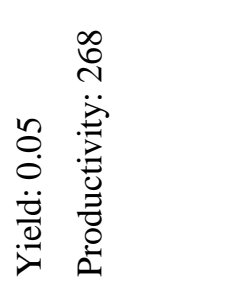 & 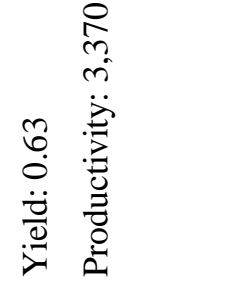 & 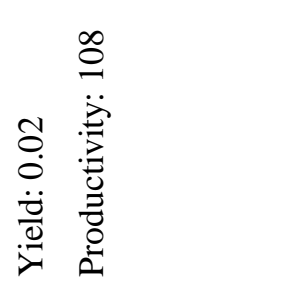 & 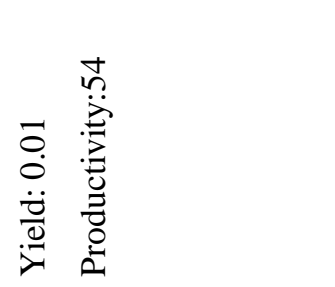 \\
\hline 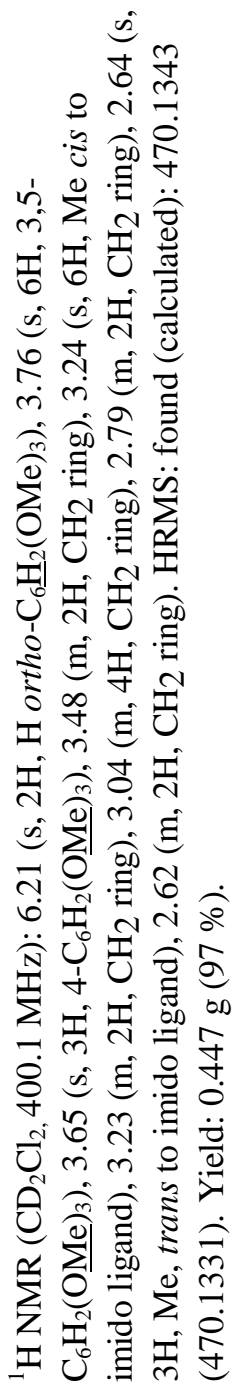 & 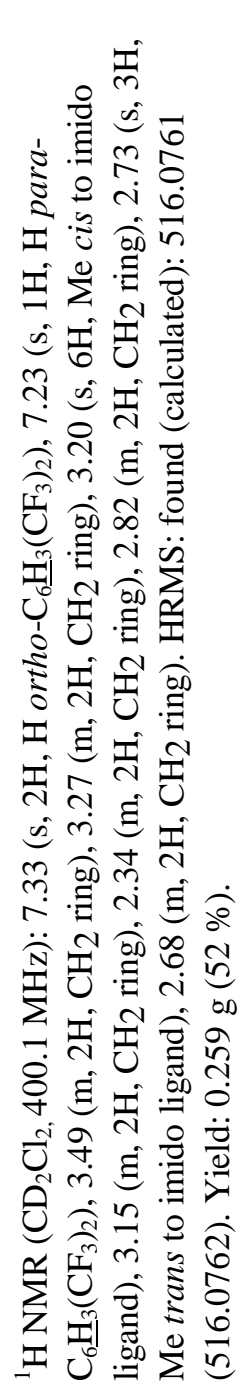 & 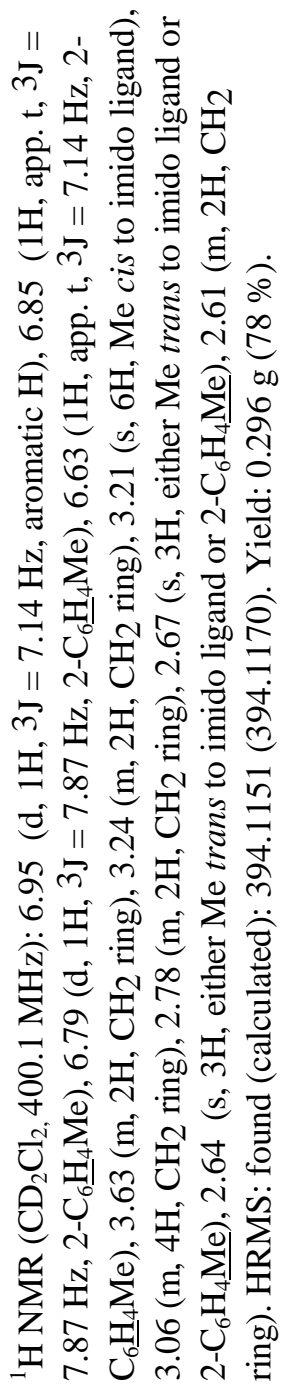 & 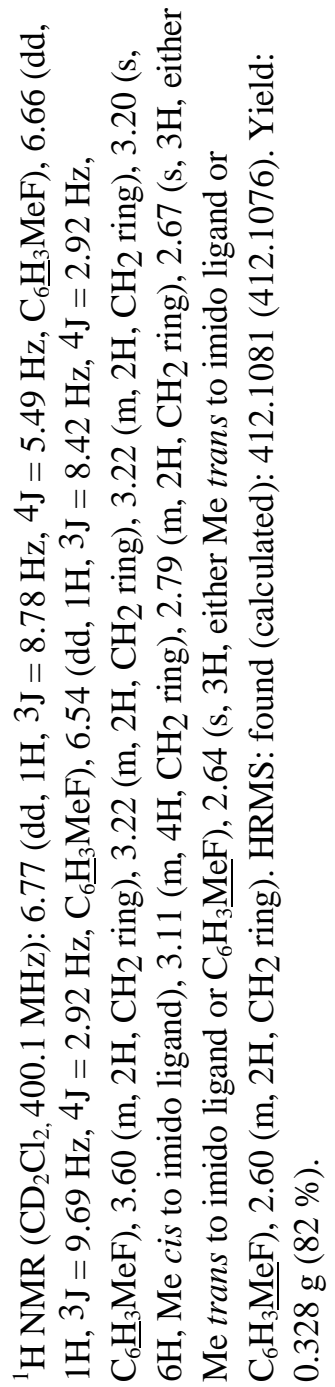 \\
\hline & & & \\
\hline 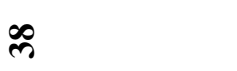 & ले & f & $F$ \\
\hline
\end{tabular}




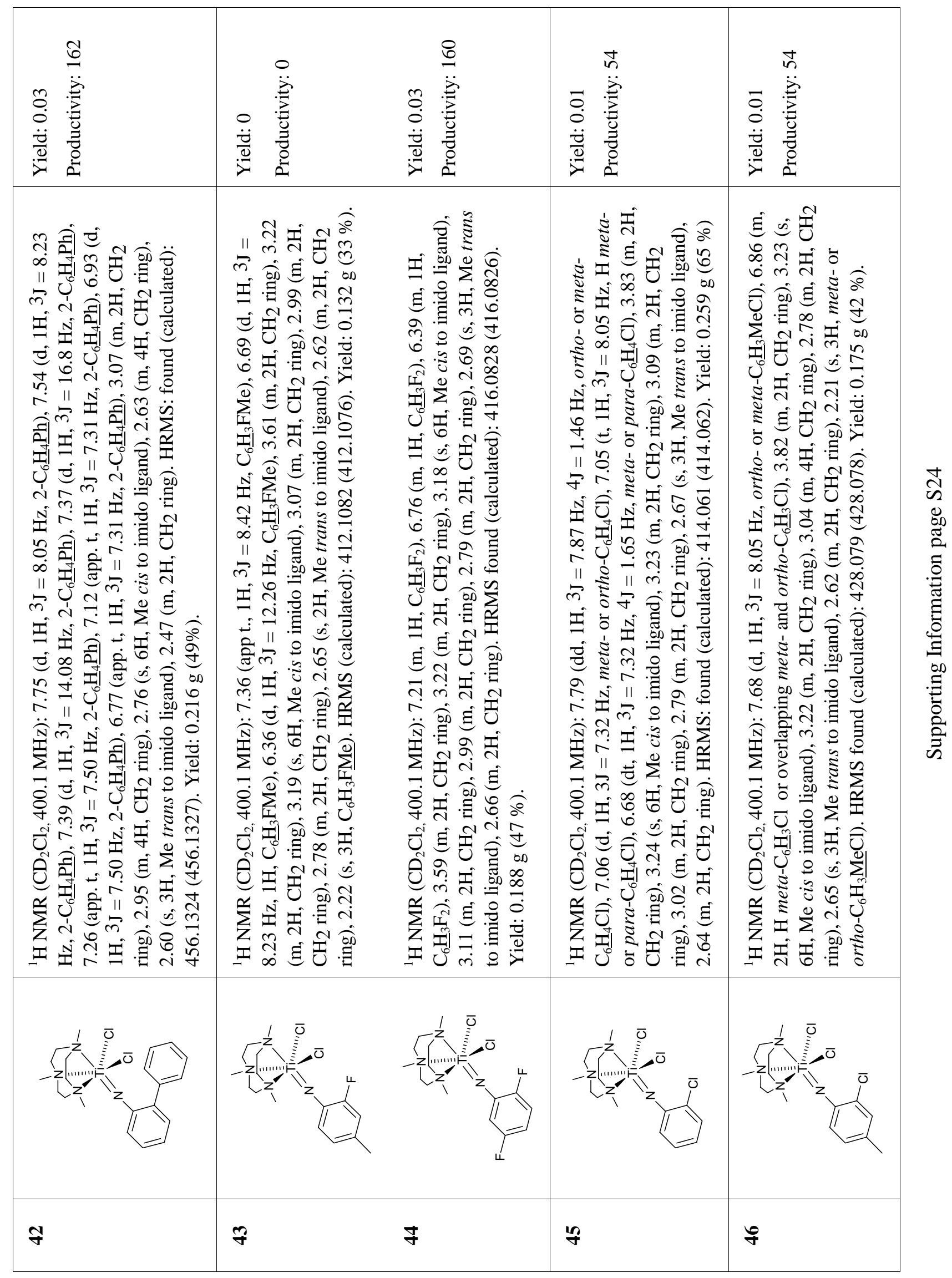









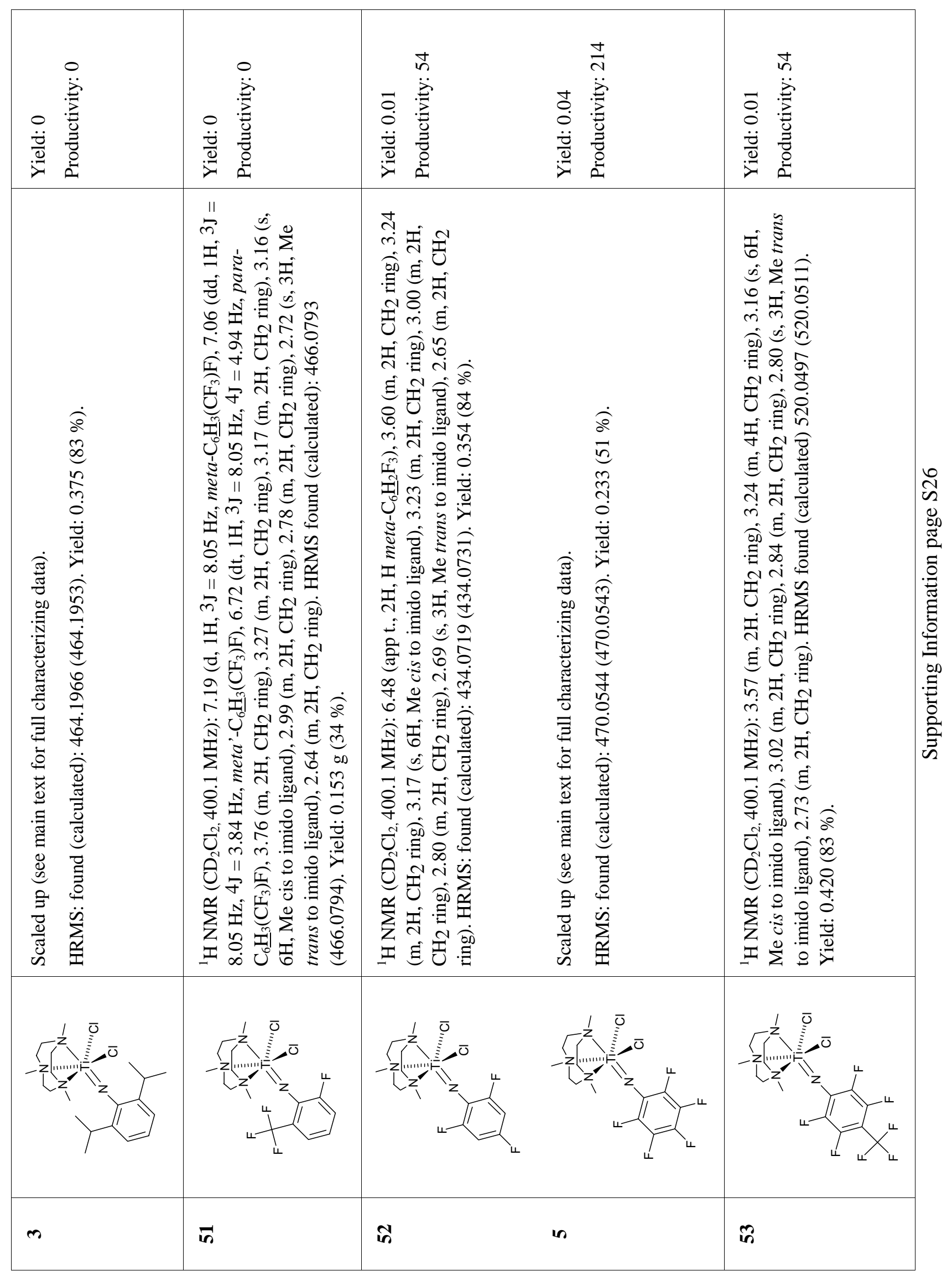




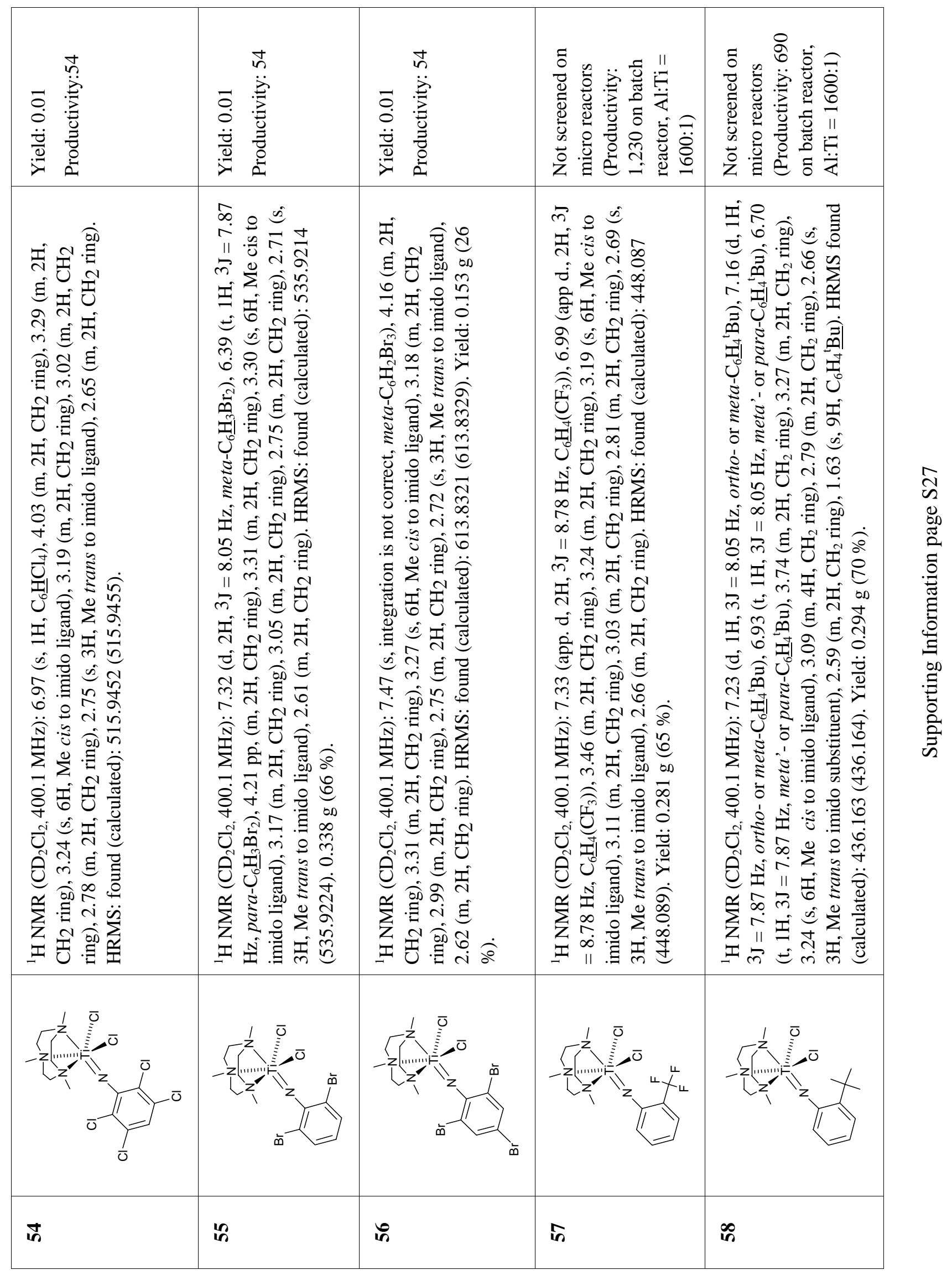




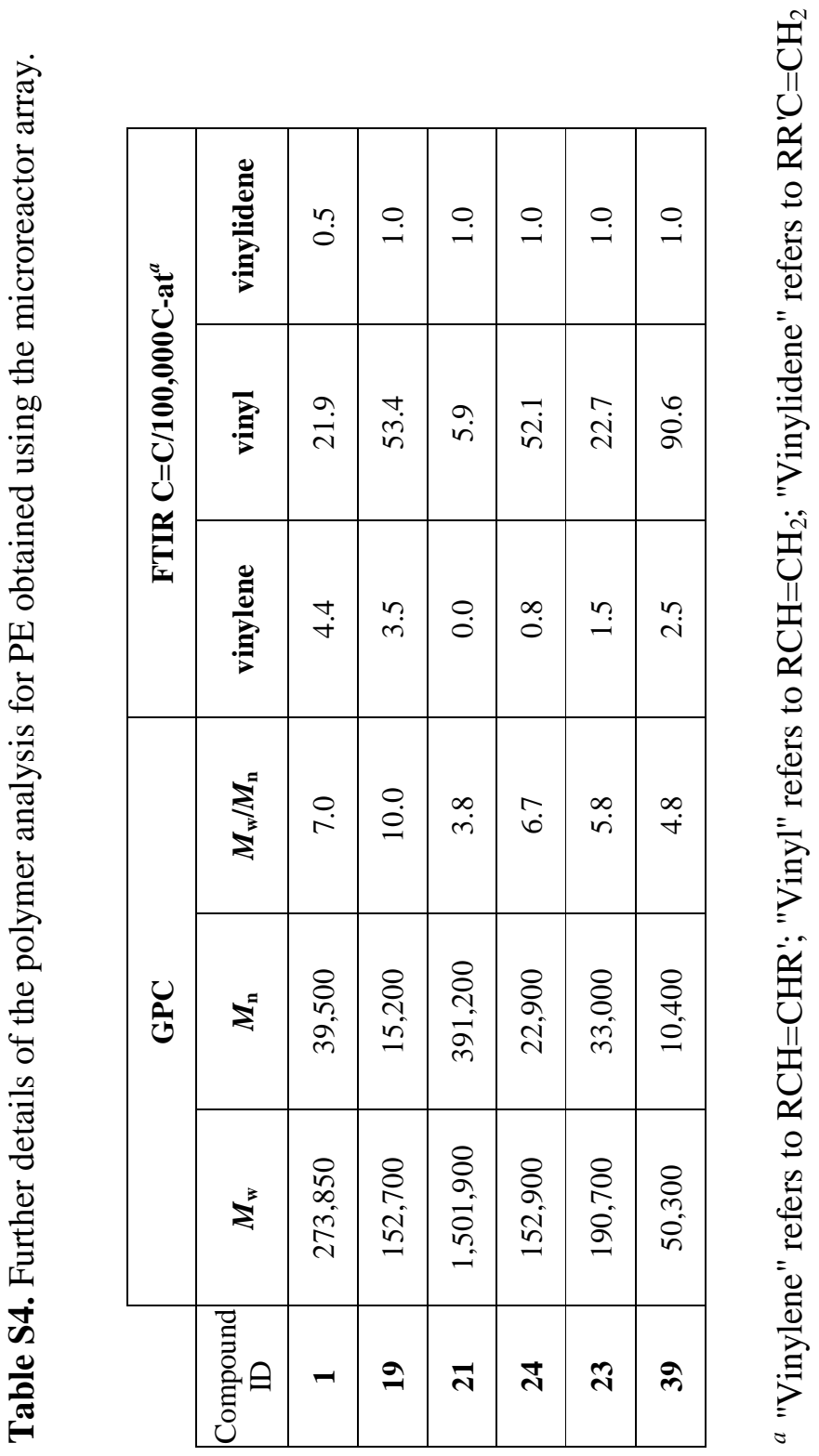




\section{Supporting Information References}

(1) Adams, N.; Bigmore, H. R.; Blundell, T. L.; Boyd, C. L.; Dubberley, S. R.; Sealey, A. J.; Cowley, A. R.; Skinner, M. E. G.; Mountford, P. Inorg. Chem. 2005, 44, 2882.

(2) Lo, H. C.; Chen, H.; Fish, R. H. Eur. J. Inorg. Chem. 2001, 2217.

(3) Bodsgard, B. R.; Burstyn, J. N. CHem. Commun. 2001, 647.

(4) de Vos, D. E.; de Wildeman, S.; Sels, B. F.; Grobet, P. J.; Jacobs, P. A. Angew. Chem. Int. Ed. 1999, 38, 980 .

(5) Grenz, A.; Ceccarelli, S.; Bolm, C. Chem. Commun. 2001, 1726.

(6) Rao, Y. V. S.; De Vos, D. E.; Bein, T.; Jacobs, P. A. Chem. Commun. 1997, 355.

(7) Fletcher, J. S.; Male, N. A. H.; Wilson, P. J.; Rees, L. H.; Mountford, P.; Schröder, M. Dalton Trans. 2000, 4130.

(8) Male, N. A. H.; Skinner, M. E. G.; Bylikin, S. Y.; Wilson, P. J.; Mountford, P.; Schröder, M. Inorg. Chem. 2000, 39, 5483.

(9) Wilson, P. J.; Blake, A. J.; Mountford, P.; Schröder, M. J. Organomet. Chem. 2000, 600, 71.

(10) Tredget, C. S.; Lawrence, S. C.; Ward, B. D.; Howe, R. G.; Cowley, A. R.; Mountford, P. Organometallics 2005, 24, 3136.

(11) Köhn, R. D.; Kociok-Köhn, G. Angew. Chem., Int. Ed. Engl. 1994, 33, 1877.

(12) Silver, G. C.; Trogler, W. C. J. Am. Chem. Soc. 1995, 117, 3983.

(13) Hoerr, C. W.; Rapkin, E.; Brake, A. E.; Warner, K. N.; Harwood, H. J. J. Am. Chem. Soc. 1956, 78, 4667. 